\title{
multiSero: open multiplex-ELISA platform for analyzing antibody responses to SARS-CoV-2 infection
}

\author{
Janie R. Byrum ${ }^{1, *}$, Eric Waltari ${ }^{1, *}$, Owen Janson ${ }^{2, *}$, Syuan-Ming Guo ${ }^{1, *}$, Jenny Folkesson ${ }^{1}$, Bryant B. Chhun ${ }^{1}$, Joanna \\ Vinden $^{2}$, Ivan E. Ivanov ${ }^{1}$, Marcus L. Forst ${ }^{1,3}$, Hongquan $\mathrm{Li}^{3}$, Adam G. Larson ${ }^{3}$, Wesley Wu ${ }^{1}$, Cristina M. Tato ${ }^{1}$, Krista M. \\ McCutcheon ${ }^{1,}$, Michael J. Peluso ${ }^{2}$, Timothy J. Henrich ${ }^{2}$, Steven G. Deeks ${ }^{2}$, Manu Prakash ${ }^{1,3}$, Bryan Greenhouse ${ }^{1,2, \bowtie}$, John \\ E. Pak ${ }^{1, \bowtie}$, and Shalin B. Mehta ${ }^{1, \bowtie}$ \\ ${ }^{1}$ Chan Zuckerberg Biohub, San Francisco, CA, USA \\ ${ }^{2}$ University of California San Francisco, CA, USA \\ ${ }^{3}$ Stanford University, Palo Alto, CA, USA \\ * equal contribution \\ @ current address: Immune-Onc Therapeutics, Inc, 795 San Antonio Road, Palo Alto, CA 94303 \\ ${ }^{\square}$ correspondence: bryan.greenhouse@ucsf.edu, john.pak@czbiohub.org, and shalin.mehta@czbiohub.org
}

\begin{abstract}
${ }_{14}$ Abstract
Serology has provided valuable diagnostic and epidemiological data on antibody responses to SARS-CoV-2 in diverse patient cohorts. Deployment of high content, multiplex serology platforms across the world, including in low and medium income countries, can accelerate longitudinal epidemiological surveys. Here we report multiSero, an open platform to enable multiplex serology with up to 48 antigens in a 96-well format. The platform consists of three components: ELISA-array of printed proteins, a commercial or home-built plate reader, and modular python software for automated analysis (pysero). We validate the platform by comparing antibody titers against the SARS-CoV-2 Spike, receptor binding domain (RBD), and nucleocapsid (N) in 114 sera from COVID-19 positive individuals and 87 pre-pandemic COVID-19 negative sera. We report data with both a commercial plate reader and an inexpensive, open plate reader (nautilus). Receiver operating characteristic (ROC) analysis of classification with single antigens shows that Spike and RBD classify positive and negative sera with the highest sensitivity at a given specificity. The platform distinguished positive sera from negative sera when the reactivity of the sera was equivalent to the binding of $1 \mathrm{ng} \mathrm{mL}{ }^{-1}$ RBD-specific monoclonal antibody. We developed normalization and classification methods to pool antibody responses from multiple antigens and multiple experiments. Our results demonstrate a performant and accessible pipeline for multiplexed ELISA ready for multiple applications, including serosurveillance, identification of viral proteins that elicit antibody responses, differential diagnosis of circulating pathogens, and immune responses to vaccines.
\end{abstract}

multiplex serology | automated analysis | accessible technology

\section{Introduction}

The 2019-2020 coronavirus disease (COVID-19) pandemic caused by the novel coronavirus SARS-CoV-2 has driven the design of numerous serological tests for antibodies against the virus. These tests have been most useful in epidemiological surveys that track the geographic and demographic distribution of virus infections (1-5). These assays have also been informative in estimating the prevalence of infection when real-time RT-PCR testing is not readily available and in identifying vulnerable populations (6). Despite the epidemiological value, serological testing is currently limited in low- and medium-income countries as shown by SeroTracker, an aggregator of global serosurveillance data on SARS-CoV-2 (7). Many of the high-performing commercially available serological assays require proprietary instruments to read the assay, and the cost of consumables, instruments, and analysis software can prohibit epidemiology surveys in resource-limited settings.

Relative to a single antigen serological test, a multiplex (i.e., a multi-antigen) serological test can provide the following advantages: a) simultaneous interpretation of the magnitude of response to multiple viral antigens and vaccine components (8-

Byrum, Waltari, Janson, Guo et al. | medR $\chi$ iv | May 7, 2021 | 1-22 
11), b) differential diagnosis of infection $(12,13)$, c) robust and quantitative measurement of antibody response, and d) increase in sensitivity due to greater coverage of immunogenic epitopes (13-19). The increased specificity and sensitivity of printed multiplex ELISA are particularly valuable when the assay is used for patient populations and cohorts with varying severity of COVID-19 or time since infection, as mild and asymptomatic infections are associated with lower antibody titers compared to severe cases $(20,21)$ and antibody response may differentially wane depending on the cognate antigen $(22)$. A panel of multiple SARS-CoV-2 antigens can also provide longitudinal measurement of antibody responses to various antigens within individuals. Longitudinal evaluation of responses to multiple antigens will be helpful in understanding which antibody responses most strongly associated with immunity to SARS-CoV-2, and how durable these responses are after infection or vaccination. In particular, multiplex serological tests will be important after widespread vaccine distribution to differentiate vaccine-induced responses from those induced by naturally acquired infection. Beyond the current COVID-19 pandemic, multiplex serological testing can be adapted for other endemic infectious agents as a tool for serosurveillance of pathogens and vaccine coverage (23).

Multiplexed ELISA follows an experimental workflow similar to conventional ELISA. Both assays consist of coating a plate with antigen(s), blocking, and overlaying an antibody-containing solution (e.g. serum). Antibodies specific to the coated antigen bind and non-specific antibodies are washed off of the surface. Biotinylated secondary antibodies recognize the Fc region of analyte antibodies, and horseradish peroxidase (HRP)-conjugated streptavidin binds to the biotinylated secondary antibodies. Alternatively, secondary antibodies can be directly conjugated to HRP. When an HRP substrate is added to the wells, the substrate reacts and produces a colorimetric signal that is proportional to the amount of analyte antibody bound to antigen in the well.

Multiplexed ELISA platforms employ a printed array of antigens (ELISA-array) (8, 9, 13, 14, 24-26), microspheres coated with antigens or antibodies (bead-based ELISA) (27-30), or a cocktail of antigens (cocktail ELISA) (19, 31-33). ELISA-array and bead-based ELISA platforms spatially separate the antigens on a surface, whereas conventional and cocktail ELISA coat the whole surface with the same antigen(s). Multiplexing with ELISA-array or bead-based ELISA offers higher specificity and sensitivity over a cocktail ELISA. Sensitivity of the cocktail ELISA falls with an increase in the number of antigens in a cocktail, which may be due to cocktail antigens blocking each other from binding to the plate (14). Commercial multiplex serology platforms include microsphere-based platforms Luminex and the substrate-printed ELISA-array platforms by RayBiotech and Scienion.

There is an outstanding need to deploy multiplex serology platforms across the world, including in low- and mediumincome countries. The commercial platforms require specialized equipment for reading the assay and analyzing the output. The analysis software is closed source and difficult to extend and automate. Open, extensible, and low-cost multiplex serology platforms can enable widespread deployment and refinement of epidemiological, longitudinal, and diagnostic serological studies.

Here we report multiSero - a multiplex ELISA platform consisting of piezo-printed ELISA-arrays (8), a plate reader, and an open-source analysis pipeline, pysero. The ELISA-array can be read using an existing plate reader, any microscope with a motorized stage, or an open plate reader (Nautilus) that we report, ergo the platform is functional with equipment accessible in many laboratories once the ELISA-arrays are printed. Pysero accepts a variety of image file formats and is agnostic to the plate reader used for data acquisition and performs better than commercial analytical platforms for printed ELISA arrays. Pysero also enables visualization and interpretation of antibody responses. The data we report were acquired on Scienion plate reader and Nautilus, a plate reader designed with low-cost components to increase accessibility to this technology (34). Thus, this platform is usable by a large range of research and public health studies.

multiSero was validated by comparing total antibody titers in 114 sera from COVID-19 RT-PCR positive individuals and 87 COVID-19 negative sera banked before the SARS-CoV-2 pandemic. We report sensitivity and specificity of classification with each antigen, with Spike and receptor binding domain (RBD) of Spike performing with the highest sensitivity, consistent with earlier reports (35-37). We report a normalization method for comparing titers across plates to eliminate the need for a standard curve on each multiplexed ELISA plate. We trained classifiers using responses to multiple antigens and found that Spike protein suffices to classify COVID-19 positive and negative sera in our cohort. Our results demonstrate a performant and accessible multiplexed ELISA analysis pipeline ready for multiple applications, including serosurveillance, identification of viral proteins that elicit antibody responses, differential diagnosis of circulating pathogens, and immune responses to vaccines. 
medRxiv preprint doi: https://doi.org/10.1101/2021.05.07.21249238; this version posted May 11, 2021. The copyright holder for this preprint (which was not certified by peer review) is the author/funder, who has granted medRxiv a license to display the preprint in perpetuity.

It is made available under a CC-BY 4.0 International license.

\section{Results}

A: Overview and components of multiSero

A Sample collection

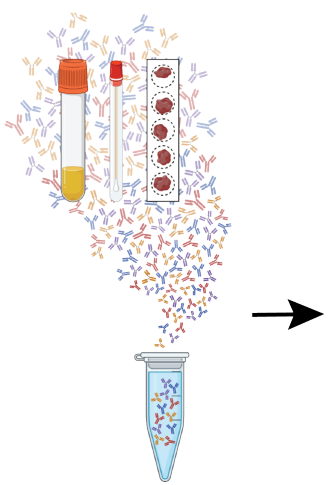

B ELISA-array

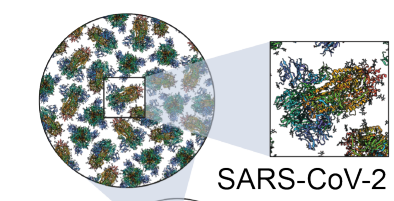

SARS-CoV-2 Spike

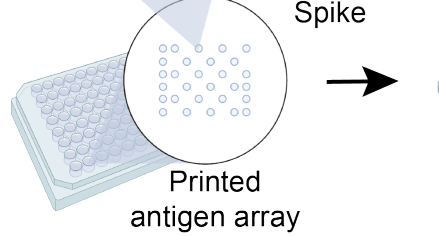

C Imaging

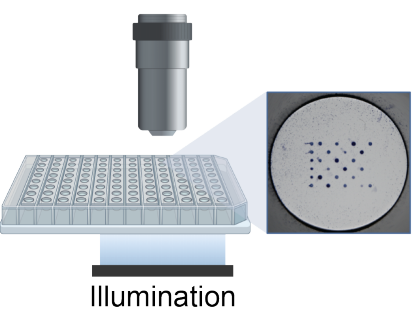

D

Pysero for multiplexed ELISA analysis
Metadata

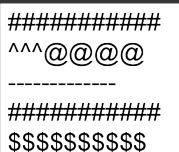

(2) Images $\rightarrow$ Optical Density

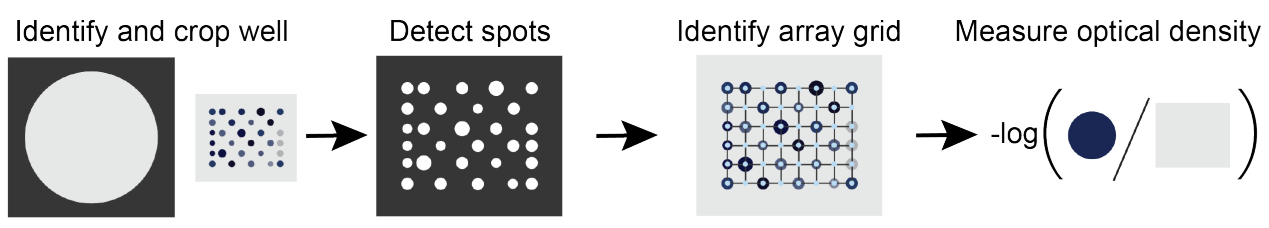

(3) Measure Antibody Titer

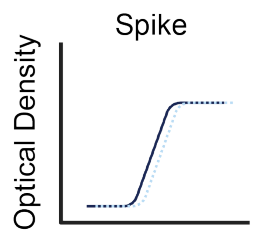
RBD

$\mathrm{N}$
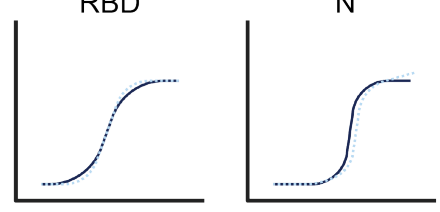

Serum Dilution

Fig. 1. Overview of multiSero pipeline: (A) Sample containing antibodies derived from serum, saliva swabs, dried blood spots, or other source are overlaid onto antigen array (B) printed by a protein arrayer such as Scienion sciFLEXARRAYER S12. Each spot in the array contains a concentration of a single protein or protein domain. (C) The ELISA is performed and then the array is imaged using Nautilus plate reader or alternative reader. (D) Pysero is used to analyze printed substrate multi-antigen ELISA-arrays. The software takes well images and a metadata file describing experimental conditions and imaging parameters as input (D1). The images are auto-cropped around the antigen array. Antigen spots are detected and a grid is registered to the spots. Optical densities are computed from the spots that align with the registered grid. Optical density is computed as the -log of the ratio of spot intensity to the background intensity (D2). Sample antibody titer against each antigen in the array can then be measured based on the ODs of the controls in the assay (D3). 
In our assay, plasma samples are added to a plate in which each well contained an array of antigens instead of a single antigen (fig. 1A). A panel of SARS-CoV-2 antigens was chosen to develop multiSero. The ELISA-array was printed using a Scienion sciFLEXARRAYER S12 (fig. 1B). The ELISA assay similar to conventional indirect ELISA was performed to enable colorimetric readout of antibody concentration. The assay is read out using a plate reader, either the SciREADER CL2 (Scienion) or Nautilus (fig. 1C; fig. S1). The plate reader used for data reported in each figure is noted in the caption. The images of ELISA-arrays were analyzed using pysero (fig. 1D) or the analysis package included with SciREADER CL2. In addition to antigen spots, ELISA array includes positive control, negative control, and fiducial markers in each well.

The Nautilus plate reader is an adaptation of the Squid microscopy platform with long-range XY stage that enables automated imaging of 96-well plates (fig. S1). The Squid platform is a performant, extensible, and open alternative with high-end microscopes with on-board computation. Pysero is a performant, extensible, and open-source alternative to commercial ELISA analysis software. Pysero provides modules that estimate antibody titers (fig. 1D, step 1) from images of the wells and metadata, as well as modules for interpretation of antibody titers. These modules are combined into pipelines for automated analysis of experiments. Nautilus and pysero together provide a complete solution for reading and analyzing developed ELISA-arrays.

As reported later, Pysero performs as well as the commercial analysis software and is independent of the plate reader (fig. 2). In addition, pysero provides machine learning classifiers that combine measurements from multiple antigens and multiple experiments. Data acquired with Nautilus is as informative as the commercial plate reader (fig. S6), the Scienion SciREADER CL2. Nautilus costs less than $\$ 1500$, is portable, and can be built with off-the-shelf parts. The bill of materials is provided in table $\mathrm{S} 1$ and the plate reader is described in the Materials and Methods.

We summarize key components of the pysero here and describe them in detail in Materials and Methods. The analysis module (fig. 1D, step 2) estimates antibody titers from the images and ELISA-array metadata. In this step, the antigen array is cropped, spots are detected, and each spot is assigned an antigen identity based on ELISA-array metadata. The detection of spots and assignment of identity rely on a grid registration algorithm that uses the fiducial spots as landmarks. The fiducial spots are deposits of antibodies whose signal intensity is invariant in the assay. Individual antigen spots are then cropped, the background around each spot is estimated, and the optical density (OD) is computed as $-\log _{10} \frac{\text { intensity }}{\text { intensity }_{\text {background }}}$. The interpretation module takes OD and experimental metadata as inputs and enables measurement and visualization of antibody concentration. Commonly used OD vs. dilution plots (fig. 1D, step 3) provide an estimate of relative antibody titers for each antigen in each sample. The absolute titer ( $\mathrm{ng} \mathrm{mL}^{-1}$ ) of antibodies can be semi-quantitatively determined by running a standard of known concentration on the plate and interpolating OD of corresponding antigen into the standard curve. The interpretation module also includes machine learning-based classifiers that pool the ODs from multiple antigens and multiple experiments to classify a given sample as COVID-19 positive or negative.

\section{B: SARS-CoV-2 ELISA-array and its analysis by pysero}

We included Spike, RBD, and Nucleoprotein (N) at two concentrations (fig. 2A) in our SARS-CoV-2 antigen array as detailed in Materials and Methods. These antigen concentrations were chosen to ensure that the antigen was present in excess of the antibodies in the sera and the dynamic range of antibody amounts led to a measurable dynamic range of OD. Future refinement of the assay would include a single optimized concentration per antigen. In addition to the SARS-CoV-2 antigens, fiducial spots consisting of biotinylated anti-kappa light chain antibodies were added to the array to facilitate grid registration and subsequent analysis. The stepwise process of grid registration using fiducials is shown in fig. 2B. The fiducial spots were printed at a constant concentration and the streptavidin-HRP recognized the fiducial spots uniformly. Anti-IgG antibody was spotted as a positive control to recognize total IgG in serum, and GFP foldon was used as a negative control, previously described by Waltari et al. (8) Introduction of artifacts is possible while performing multiplexed ELISA, and common artifacts include comets and debris. Both comets and debris are present in the representative image read by a SciREADER CL2 in fig. 2C. The layout and spacing of the antigen array was such that experimental artifacts, such as smearing of spots (comets, example in fig. 2C), would minimally affect the analysis of adjacent spots.

We compared the performance of the SciREADER CL2 software and pysero in recognizing the spots in the array. The SciREADER CL2 failed to identify four spots that were partially obfuscated by comets, whereas pysero detected these spots 
medRxiv preprint doi: https://doi.org/10.1101/2021.05.07.21249238; this version posted May 11, 2021. The copyright holder for this preprint (which was not certified by peer review) is the author/funder, who has granted medRxiv a license to display the preprint in perpetuity.

It is made available under a CC-BY 4.0 International license .

A

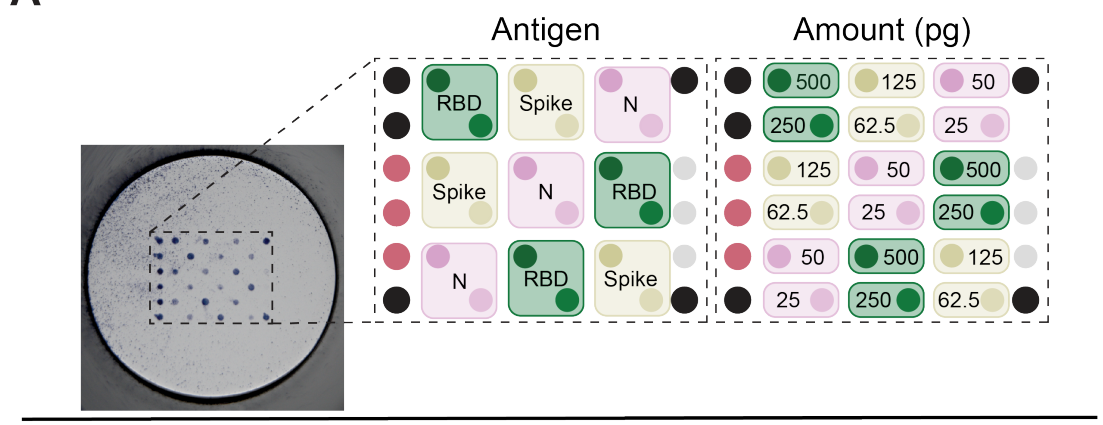

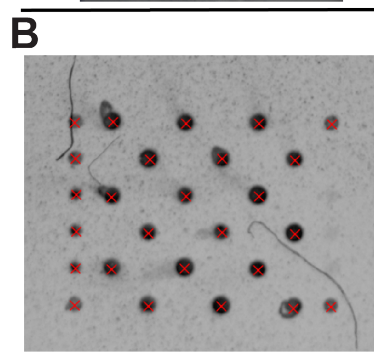

$\mathrm{x}$ Spot detection

C

Original Image

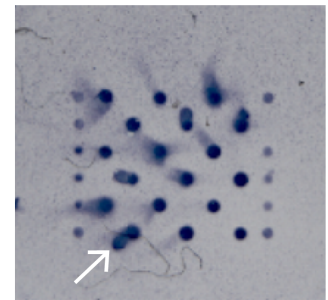

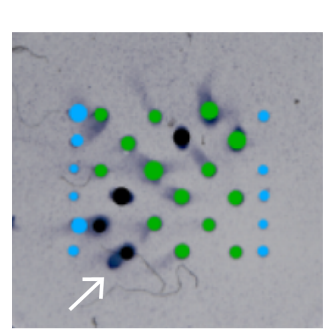

- Not found

- Fiducial spots

- Positive spot value

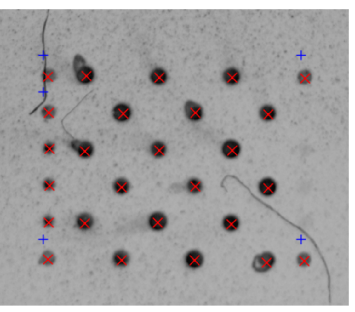

$x$ Spot detection

+ Initial fiducial spot guess

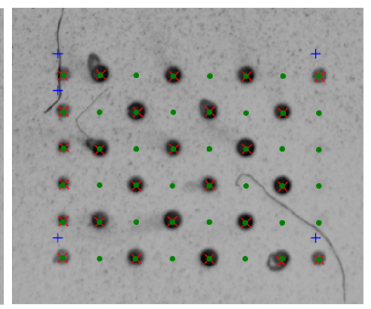

$\mathrm{x}$ Spot detection

+ Initial fiducial spot guess

- Final grid registration

Pysero detection

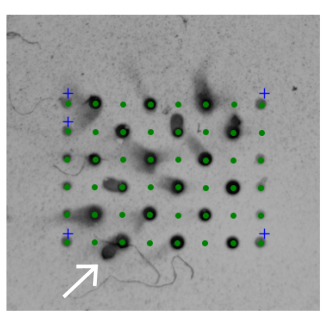

- Not found

+ Initial fiducial spot guess

- Final grid registration

Fig. 2. ELISA-array for SARS-CoV-2 and its analysis with pysero. (A) Image of well containing Scienion-printed antigen array. Array layout shows the relative locations of SARS-CoV-2 antigens included in the array: receptor binding domain of spike-RBD (RBD; green), spike (ochre), and nucleoprotein (N; pink). Each antigen was spotted at two concentrations which are indicated in the right panel of the layout. Black spots represent anti-kappa-biotin fiducials, mauve spots represent anti-lgG Fc, and grey spots represent GFP foldon. (B) Pysero first detects center points of all spots in the cropped image (red x). Fiducial positions (blue + ) are initialized based on detected spot coordinates. Coordinate transformation that registers the initial fiducials with detected fiducials is estimated using particle filtering. A grid containing all spot locations (green -) in the antigen layout is then transformed onto the image using the estimated coordinate transformation. The coordinates of each spot are then used to crop individual spots and extract their OD. (C) Comparison of Scienion analysis and pysero spot detection. Original image of a well imaged by SciREADER CL2 includes comets (white arrow) and fiber-like debris. Center image is the output of Scienion spot detection and analysis. A black spot indicates an analyte-containing spot was not found at the location, green spots indicate positive spot value, and blue spots indicate the location of fiducial spots. The size of the spot indicates the area analyzed by Scienion. Right image is the output of pysero. Green dots indicate registered grid positions (size of marker unrelated to area analyzed), blue crosses indicate initialized fiducial spots. 
(fig. 2C). Furthermore, we compared spot intensities, background intensities, and ODs derived from the SciREADER CL2 software and pysero, and found similar trends (fig. S2). Pysero was more robust to comets relative to the SciREADER CL2 software in two respects: spot detection and background estimation. The SciREADER CL2 software detected spots that were larger than the central spot when the spot had a comet which resulted in the inclusion of background pixels in the spot region. Including background pixels in the spot region results in higher intensity, and thus lower OD. The SciREADER CL2 background estimation was also affected by comets in that it estimated background from the pixels surrounding detected spots assuming circular spot shape. Therefore, the SciREADER CL2 analysis platform underestimated the background intensity when there was a comet, and this resulted in lower OD. Additionally, the Scienion analysis platform is only capable of analyzing images acquired using the SciREADER CL2, prohibiting a comparison of Nautilus images and SciREADER CL2 images analyzed by the SciREADER CL2 software.

We evaluated whether the comets bias the ODs measured with pysero by comparing the duplicate spots, i.e., spots from the wells that contained the same antigens and same serum (fig. S3). We find that the presence of comets does not cause observable bias or variance in ODs measured with pysero. In sum, pysero enables more robust analysis of antibody titers than the commercial analysis software available with SciREADER CL2.

\section{C: Limits of detection and quantitative analysis}

Each antigen on the array has an independent lower limit of detection which is influenced by the amount of non-specific serum protein binding to each antigen in the sample. We determined the lower limit of antibody detection for each antigen in human serum by evaluating sera from two cohorts: a group of individuals from the Long-term Impact of Infection with Novel Coronavirus (LIINC) cohort, all of which tested positive for SARS-CoV-2 by qRT-PCR test, and sera acquired from a blood bank that was banked before SARS-CoV-2 was circulating in the population. We termed the serum pool from the LIINC cohort as "Positive Pool", and the serum pool from the pre-pandemic sources as "Negative Pool".

We first evaluated which of the two amounts of Spike, RBD, and N proteins are suitable for detection of antibody titers in SARS-CoV-2 positive and negative sera in our cohort. ELISA with dilution series of the Positive Pool and the Negative Pool (fig. S4) showed that $50 \mathrm{pg}$ of $\mathrm{N}\left(1 \mathrm{~nL}\right.$ spotted at $\left.50 \mu \mathrm{g} \mathrm{mL}{ }^{-1}\right), 250 \mathrm{pg}$ of RBD $\left(1 \mathrm{~nL}\right.$ spotted at $\left.250 \mu \mathrm{g} \mathrm{mL} \mathrm{m}^{-1}\right)$, and $62.5 \mathrm{pg}$ $\left(1 \mathrm{~nL}\right.$ spotted at $62.5 \mu \mathrm{g} \mathrm{mL} \mathrm{mL}^{-1}$ ) of Spike transition from not being detectable at low concentrations of Positive Pool to being saturated at high concentrations of the Positive Pool while these amounts of antigen remain unsaturated by the Negative Pool at all serum concentrations. This data clarified that using the lower of the two amounts we spotted for each antigen suffices to discriminate antibody titers between positive and negative sera and clarified appropriate serum dilutions for accurate antibody titering given the amount of antigen spotted.

Results for antibody responses against $250 \mathrm{pg}$ of spotted RBD are shown in fig. 3A-D and all other antigens are summarized in table S2. To determine the relative range of antibody responses to each antigen, multiplexed ELISA was performed using a dilution series of the Positive Pool and Negative Pool (fig. 3B). For a given serum dilution, a sample is positive for antibodies against a SARS-CoV-2 antigen if the unknown sample's OD is three standard deviations above the mean Negative Pool OD at that dilution.

Quantitative measurement of antibody titer to each antigen in the array varies based on antibody affinity and concentration. To estimate the antibody titers specific to RBD and Spike, we generated a standard curve with a monoclonal antibody (mAb) CR3022 (38), which recognizes a conserved epitope of RBD of SARS-CoV-2 and SARS-CoV. The CR3022 titration was performed using the Negative Pool as a diluent to match the matrix found in human sera. Figure 3B shows OD vs 7 serial dilutions of the mAb CR3022 in the range $1-0.00024 \mu \mathrm{g} \mathrm{m} \mathrm{m}^{-1}$ (fig. 3B; grey points). We also show 4 parameter logistic regression of this data (fig. 3B; grey points), which allows us to interpolate the reactivity of antibodies in sera from the measured ODs (fig. 3B; inset). For $250 \mathrm{pg}$ of spotted RBD, the data indicates: a) the lowest concentration of CR3022 in Negative Pool $\left(0.24 \mathrm{ng} \mathrm{mL}^{-1}\right)$ has reactivity equivalent to the Negative Pool alone (fig. 3B), and b) the Negative Pool on average has reactivity equivalent to $0.4 \mathrm{ng} \mathrm{mL}^{-1}$ of CR3022. Antibody responses against other antigens are shown in table $\mathrm{S} 2$. Using this assay, we can qualitatively determine whether individual samples contain antibodies against specific antigens and also approximate the amount of sample reactivity by equating OD to the equivalent of mAb CR3022 recognizing RBD at a known concentration and binding affinity. 
medRxiv preprint doi: https://doi.org/10.1101/2021.05.07.21249238; this version posted May 11, 2021. The copyright holder for this preprint (which was not certified by peer review) is the author/funder, who has granted medRxiv a license to display the preprint in perpetuity.

It is made available under a CC-BY 4.0 International license .

A

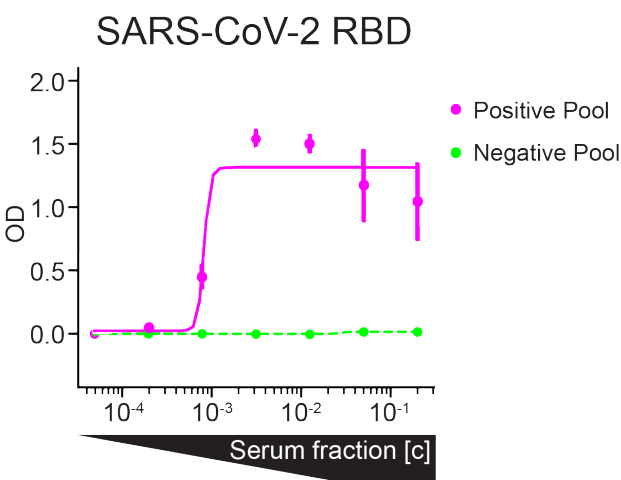

C

\section{SARS-CoV-2 RBD}

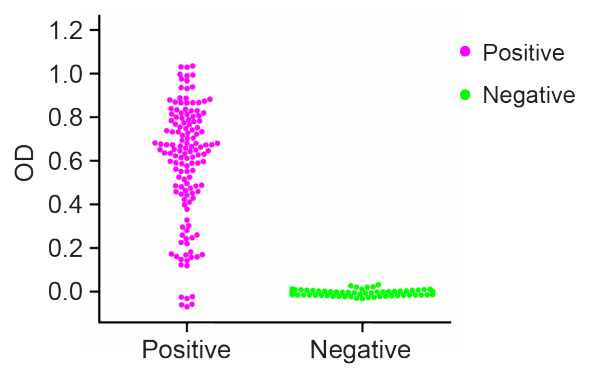

B

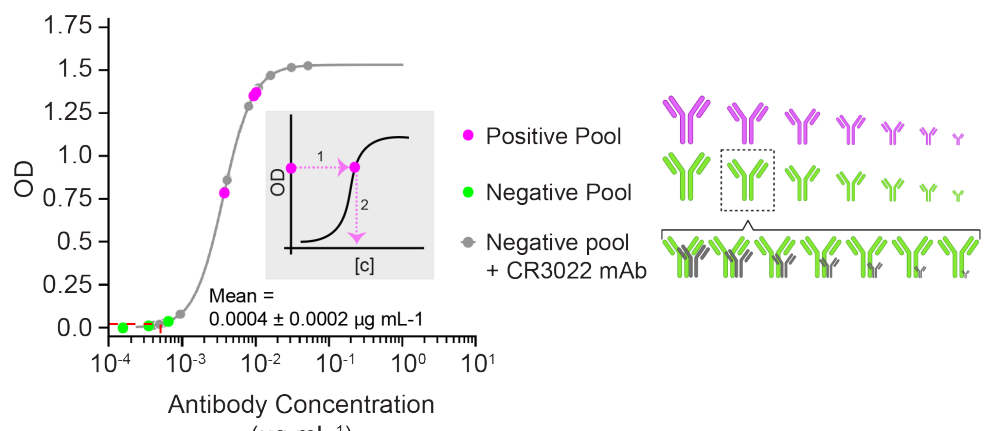

$\left(\mu \mathrm{g} \mathrm{mL} \mathrm{L}^{-1}\right)$

D

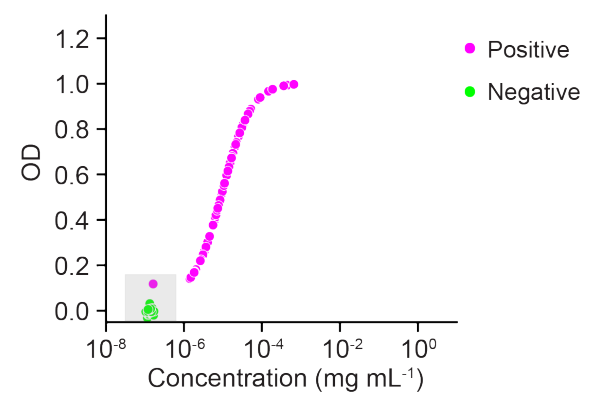

Fig. 3. Determining limits of detection in the presence of complex background and validation by SARS-CoV-2 epidemiology survey. (A) ODs denoting antibody response against RBD antigen spot (250 pg). Serial dilution of sera pooled from RT-PCR-positive cohort (magenta solid line) and a pooled RT-PCR-negative cohort (green dashed line). (B) Concentration of antibodies against RBD spotted at 250 pg present in pooled positive sera (magenta points) and pooled negative sera (green points) at $1 / 200$ dilution. Grey curve and points denote standard curve (OD vs known antibody concentration) obtained from serial dilution of mAb CR3022 in the $1 / 200$ diluted Negative Pool. The antibody schematic at right illustrates 7-point serial dilutions of the pooled positive sera (magenta), the pooled negative sera (green), and the mAb CR3022 (grey) in the 1/200 diluted Negative Pool. The ODs corresponding to the Positive Pool and Negative Pool were interpolated into the standard curve, and the resulting antibody concentration of the unknowns can be read out from the x-axis using the fit of the standard curve. The mean antibody concentration of the Negative Pool (1/200 serum dilution) is indicated on the plot (mean $=0.0004 \pm 0.0002 \mu \mathrm{g} \mathrm{mL}{ }^{-1}$; red dashed line), and the lower limit of detection for a positive result is 3 standard deviations above this mean. Data for panels (A-B) were acquired using SciREADER CL2. (C) Range of ODs of RT-PCR-positive individuals (magenta) and RT-PCR-negative individuals (green) from a small episurvey using samples from the LIINC cohort. Each point represents one triplicate for each antigen. (D) ODs from individual serum samples interpolated into mAb CR3022 standard curve. Sera are colored according to their RT-PCR-positive or RT-PCR-negative status. An OD that is outside of the range of ODs corresponding to the standard curve cannot be quantified. Points that are lower than the lowest $\mathrm{mAb} C R 3022$ concentration are plotted by $\mathrm{OD}$ and arbitrarily at $10^{-7} \mathrm{mg} \mathrm{mL}^{-1}$. Data for panels (C-D) were acquired with Nautilus. 
Multiplexed ELISA was performed on the individual serum samples to determine the range of antibody responses against SARS-CoV-2 (fig. 3D). We set the positivity threshold for specific antibodies against RBD in this assay at $1.1 \mathrm{ng} \mathrm{mL}^{-1}$, i.e., three times the standard deviation of the mean binding reactivity of the Negative Pool. Three samples from individuals with positive qRT-PCR results had lower antibody concentrations against RBD than the lowest concentration on the mAb standard curve, $1.6 \mathrm{ng} \mathrm{mL}^{-1}$ (fig. 3D; grey inset). These samples had low responses to all antigens in the array, suggesting these individuals had sub-optimal antibody responses to this infection.

\section{Normalization methods for large-scale multiplexed ELISA data}

One approach to quantitative analysis of reactivity is to incorporate a set of standard antibodies as we reported. But, incorporating a set of standards to generate a standard curve per antigen in the array each time multiplexed ELISA is performed is reagent-heavy and labor-intensive. The use of standard curves also reduces the number of samples that can be run per plate. An attractive alternative is to normalize the ODs of each experiment to the experiment that included the standards. ELISAarray provides fiducials and positive controls that facilitate such data normalization. Proper normalization is also necessary for pooling data from across the experiments for statistical analysis, e.g., training more accurate diagnostic classifiers.

We explored normalization of multiplexed ELISA OD values to enable pooling of data across plates without the need to run a standard curve per plate. We explored 3 normalization methods: The ODs of spots in duplicate plates were normalized with the ODs of fiducial spots (biotinylated anti-kappa light chain antibodies that are detected by the streptavidin-conjugated secondary antibody) per plate, the ODs of the anti-IgG Fc spots per plate, or the ODs of anti-IgG Fc spots per well (fig. S5). Each spot location in the array was compared to the spot with the same array location in a duplicate plate. Without normalization, duplicate plate datasets exhibited low bias and noise. After normalizing by the anti-kappa light chain fiducial signal present in each plate, bias and noise were marginally reduced for one out of three datasets, with bias $=-0.074$ and noise $=0.108$ without normalization compared to bias $=-0.023$ and noise $=0.082$ after normalization. Normalizing by anti-IgG Fc signal present in each plate resulted in a subtle reduction in bias and noise consistently in all 3 datasets. Normalizing the OD values in each well by the anti-IgG Fc signal present in the same well decreased the bias in 2 out of 3 datasets but considerably increased the noise in each dataset. The best normalization method provided only a small improvement over no normalization. These experiments were performed using the same reagent lots and in a short span of time. ELISA-array data accumulated over weeks or months and using multiple lots of reagents are expected to have larger variability and can benefit from these normalization procedures.

\section{D: Sensitivity and specificity of SARS-CoV-2 multi-antigen ELISA}

To determine the best antigen or antigen set for discriminating positive and negative sera, we trained gradient boosting tree classifiers using RT-PCR status of the sera as the ground truth. We used data from 189 sera (106 positive, 83 negative). All sera were assayed at least in duplicate, some of them in quadruplicate and were spread over 6 different plates. The robustness of trained classifiers was evaluated using receiver operating characteristic (ROC) curve and the area under the curve (AUC). Sensitivity of the assay at $95 \%$ and $99 \%$ specificity using single antigens are reported for each antigen in table S2 (N=507). In fig. 4 and fig. S6, we report ROC curves and sensitivity at $95 \%$ specificity.

We evaluated if the choice of plate reader affects the performance of the assay. We used pysero to compare sensitivity and specificity of classification of the COVID-19 positivity of samples by analyzing the data acquired with SciREADER CL2 and Nautilus (fig. S6).The equivalent classification performance illustrates that the data acquired with Nautilus is as informative as commercial solutions and pysero can be used with any plate reader.

To test if incorporating information from multiple antigens improves the classification performance, we trained a gradient boosting tree classifier on $60 \%$ of the SARS-CoV-2 positive and negative serum data (training and validation sets) in the cohort and performed classification on the remaining $40 \%$ of the dataset (test set). The ROC curves for each antigen and the classifier were generated on the test set ( $\mathrm{N}=189)$ (fig. 4). The ROC curve reports the true positive rate (sensitivity) and false positive rate (1 - specificity) as a function of the OD threshold (for single antigens) or probability threshold (for the classifier) used to classify the serum samples. RBD and spike show similar performance (97.7-97.8\% sensitivity) for both spotted concentrations. Nucleoprotein shows worse performance (95.5\% sensitivity at 95\% specificity) (fig. 4A). Using this analysis, users can select OD thresholds depending on the metric (i.e. sensitivity or specificity) more appropriate for their application. For example, 
medRxiv preprint doi: https://doi.org/10.1101/2021.05.07.21249238; this version posted May 11, 2021. The copyright holder for this preprint (which was not certified by peer review) is the author/funder, who has granted medRxiv a license to display the preprint in perpetuity.

It is made available under a CC-BY 4.0 International license.

A

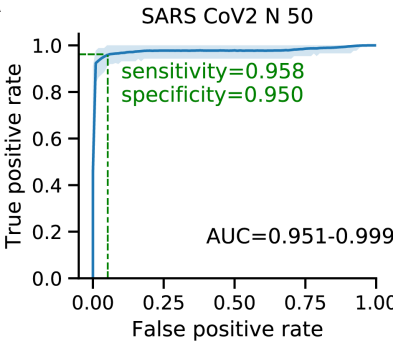

B

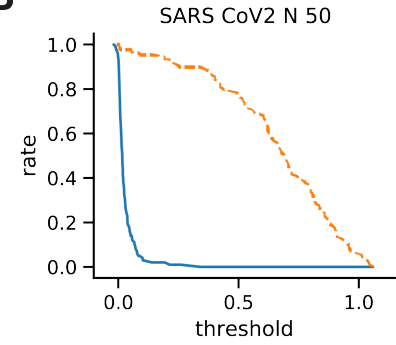

SARS CoV2 RBD 250

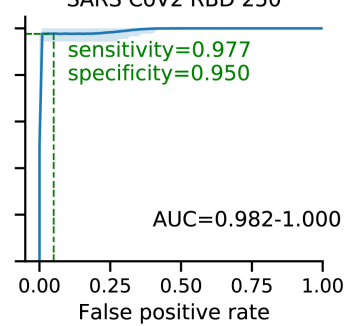

False positive rate

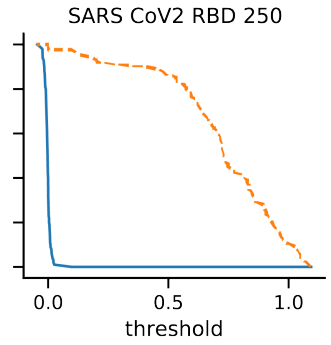

SARS CoV2 spike 62.5

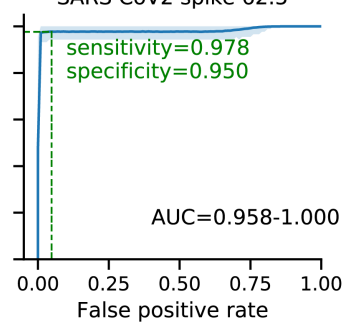

SARS CoV2 spike 62.5

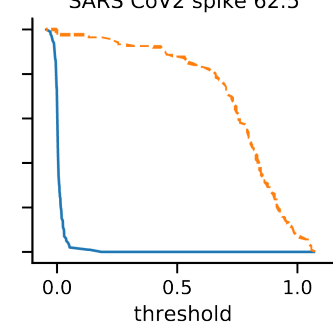

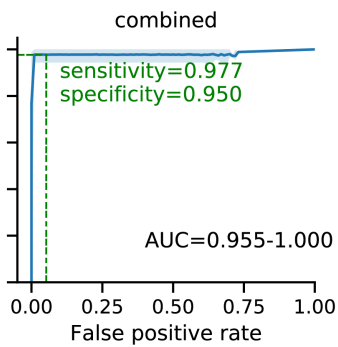

combined

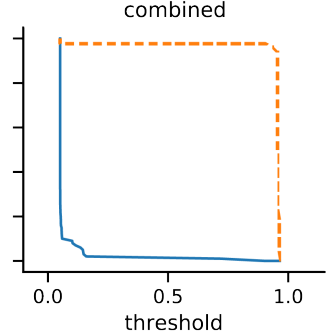

category

_ False positive rate --- True positive rate

Fig. 4. Performance of classifiers that use single and multiple antigens to classify positive and negative sera. (A) ROC curves (blue line) for classifiers that use single antigens (left to right: nucleoprotein, RBD, and spike) and a classifier that combines information from multiple antigens. Blue shades represent $95 \%$ confidence intervals of ROC curves. For each antigen, the sensitivity (True positive rate (TPR)) at 95\% specificity (1 - false positive rate (FPR)) is denoted (green dashed line) on the curve. $95 \%$ confidence interval of area under the ROC curve (AUC) is reported below each curve. (B) TPR (orange dashed line) and FPR (blue line) represented as a function of the OD threshold (for single antigens) or probability threshold (for the classifier) used to classify positive and negative sera. ODs were normalized by mean anti-IgG Fc signal per plate. 189 sera (106 positive, 83 negative) were assayed at least in duplicate, some of them in quadruplicate, spread over 6 different plates. Data for fig. 4 were acquired with Nautilus. 
first-pass surveys to screen for potential positives in a large cohort may tolerate a higher probability for false alarm to capture all positives in the initial screen in lieu of the highest possible sensitivity. Figure 4B illustrates how true positive rate and false positive rate change as the threshold value varies.

The classifier that uses ODs from all antigens performed with $97.7 \%$ sensitivity at $95 \%$ specificity, which is comparable to the best performing single antigens (RBD and Spike). Examining the images and ODs of the false negative classifications showed low OD across all antigens in both replicates, which clarifies why incorporating information from all antigens did not help correct these false negative samples. On the other hand, all false positive serum samples showed relatively high OD values for some antigens but only in one of the duplicates, indicating these false-positive samples most likely result from high background that randomly occurs during the assay, and could be eliminated by including more replicates.

\section{Discussion}

multiSero platform provides commercial-grade sensitivity and specificity with an in-house surface-printed antigen array, open source analysis software (pysero), and open source plate reader (Nautilus). Our multiplexed ELISA assays of SARS-CoV-2 antigens illustrate that this open pipeline provides performance comparable to commercially available platforms and provides features that are not available in commercial platforms. Commercially available printed-substrate ELISA-arrays either require a proprietary plate reader and analysis software or they are compatible with laser scanners and software used for protein microarrays (Raybiotech, Atlanta, GA; Quansys, Logan, UT; Scienion, Berlin, Germany; Abcam, Cambridge, MA; Meso Scale Diagnostics, Rockville, Maryland). Because pysero is agnostic to the image source, it can also be used to analyze data from a variety of surface-printed array products including those that use fluorescence as readouts. Here we have tested pysero with data acquired using Scienion's SciREADER CL2 and the Nautilus plate reader.

The described SARS-CoV-2 multi-antigen array is useful as an epidemiology survey tool as it has increased throughput compared to conventional ELISA by increasing the number of analytes per plate. The in-well biotinylated anti-kappa light chain spots, GFP foldon spots and anti-IgG Fc spots function as internal controls and decrease the sample volume needed per assay. The sample size of this study is small; however, sensitivity at $100 \%$ specificity will increase as this array is more routinely used for epidemiological surveillance.

Accuracy of analyzing the surface-printed array can be improved through minimization of artifacts such as comets. Comets are smearing of spotted antigen:antibody complexes and are true signal (fig. S3), so it is important to integrate the signal at the registered center of the spot and the comet for the most quantitative result. Currently pysero only extracts OD surrounding the registered center of the spot. Comets are common to surface-printed protein arrays, and one hypothesis is that comets form when the surface is not completely dry before performing the ELISA. In our hands, waiting up to 30 minutes for the plate to equilibrate at room temperature before the experiment modestly reduced comets, suggesting alternative sources of comets. In anticipation of comets, we implemented 2 practical solutions to reduce effects of comets regardless of their source. First, we designed an antigen array layout in a checkerboard pattern so that comets would not overlap with nearby spots and interfere with OD extraction. Second, each antigen is in triplicate within the array and the layout is such that each triplicate location is distant from the other triplicates. Future iterations of this assay may explore alternative array technology to ameliorate comet troubleshooting. For example, the surface of the plate, plate porosity, or the formulation of the protein stock for printing could be altered to prevent mobility of the spot, or microfluidic devices could be used to control flow of sera and reagents as well as isolate antigen reservoirs.

Multiplexed ELISA and open-source software have high potential for surveying exposure to multiple viruses in low-resource regions with endemic infectious disease. Many infectious diseases (not only viral) manifest with nonexclusive symptoms, and a multiplexed ELISA-array with antigens representing circulating pathogens can be used to simultaneously evaluate the prevalence of multiple pathogens. The approach of using surface-printed protein arrays and open source analysis tools can be adapted for multiplexed detection of pathogens by printing pathogen-specific antibodies, instead of antigens.

Non-contact arrayers can be purchased for $\$ 100,000-300,000$. The printing technology is the most expensive aspect of the pipeline, and access to the antigen array printer is a limiting factor in wide-spread deployment of multiplexed ELISA to resource-poor regions. Advances in bioprinting technology enable the possibility of an open arrayer (39). Partnerships with 
non-governmental organizations and multi- or bi-lateral agencies with global health missions can provide support for hardware or cold chain storage of surface-printed arrays. Alternatively, arrays with more intrinsic stability and portability can be devised. Two such examples are the origami-inspired foldable paper protein arrays or microfluidic devices (40-42), which would also rid the current platform of analysis challenges due to comets.

A combined classifier was trained on responses to all array antigens using sera from confirmed SARS-CoV-2 positive individuals and negative sera banked prior to the COVID-19 pandemic. We chose the gradient-boosting framework since it enables interpretation of feature importance. We find that there is no advantage in classifying the positive/negative cases when responses to multiple antigens are combined, because the single best antigen, Spike spotted at $62.5 \mathrm{pg}$, provided high discriminatory power. The combined classifier may increase the specificity and sensitivity for multiplex assays that have less specific responses. For example, less expensive serology pipelines, such as ReScan (11) employ linear peptides that interact with lower specificity than the folded proteins. Our approach of building an interpretable classifier may improve the specificity and sensitivity of such assays. To our knowledge, other analysis programs for multi-antigen ELISA currently do not offer combined analysis of multiple antigens in order to make positive/negative calls of a sample.

In summary, multiSero provides a performant and scalable pipeline consisting of open tools that can enable multiple diagnostic and epidemiological studies: serosurveillance, differential diagnosis of circulating pathogens, detection of viral proteins, and immune responses to vaccines.

\section{Materials and Methods}

\section{A: Recombinant protein production}

Plasmids encoding SARS-CoV-2 Spike (S) ectodomain or Receptor Binding Domain (RBD)36,41 were transiently transfected into suspension Expi293 cells at 1-1.5 L scale in 2.8 L Optimum Growth Flasks (Thomson Scientific), following the manufacturer's guidelines. Three days after transfection, cell cultures at $>75 \%$ viability were centrifuged at $500 \mathrm{~g}$ for $30 \mathrm{~min}$, followed by filtration of the supernatant using a $0.45 \mathrm{um} \mathrm{NalGene} \mathrm{Rapid} \mathrm{Flow} \mathrm{filter} \mathrm{unit.} \mathrm{The} \mathrm{supernatant} \mathrm{was} \mathrm{adjusted} \mathrm{to} \mathrm{pH} 7.4$, and directly loaded onto a $5 \mathrm{~mL}$ HisTrap Excel column pre-equilibrated with $20 \mathrm{mM}$ sodium phosphate, $500 \mathrm{mM} \mathrm{NaCl}, \mathrm{pH} 7.4$ using an AKTA Pure. Captured proteins were washed with 60 column volume (CV) of (20 mM sodium phosphate, $500 \mathrm{mM}$ $\mathrm{NaCl}, 20 \mathrm{mM}$ imidazole, $\mathrm{pH} 7.4)$ and eluted with $10 \mathrm{CV}$ of (20 mM sodium phosphate, $500 \mathrm{mM} \mathrm{NaCl}, 500 \mathrm{mM}$ imidazole, $\mathrm{pH}$ 7.4). Eluted proteins were buffer exchanged into PBS using either 3kDa MWCO (for RBD) or $100 \mathrm{kDa}$ MWCO (for Spike) Amicon concentrators, supplemented with $10 \%$ glycerol, and filtered prior to storage at $-80^{\circ} \mathrm{C}$. Protein stability after freeze thaw was confirmed by analytical SEC-MALS.

A codon-optimized His tagged SARS-CoV-2 Nucleocapsid (N) gene (43) was synthesized and cloned into a pET-28 vector by Twist Bioscience (San Francisco, CA, USA). The expression plasmid was transformed into T7Express bacterial expression cells (New England Biolabs, Ipswich, MA) and a single colony was used to grow $10 \mathrm{~mL}$ of overnight culture in LB/Kanamycin, which was then used to inoculate $1 \mathrm{~L}$ of $\mathrm{LB} / \mathrm{Kanamycin}$. The $1 \mathrm{~L}$ culture was shaken at $37^{\circ} \mathrm{C}, 200 \mathrm{rpm}$ until the $\mathrm{O}$. $\mathrm{D}$. 600 reached 0.6 . The temperature of the culture and incubator were lowered to $25^{\circ} \mathrm{C}$ and protein expression was induced by addition of $0.5 \mathrm{mM}$ Isopropyl- $\beta$-D- thiogalactoside (IPTG). After $20 \mathrm{~h}$, the bacterial cells were isolated by centrifugation and the pellet resuspended with Buffer 1 ( $50 \mathrm{mM}$ phosphate, $\mathrm{pH} 8.0,1 \mathrm{M} \mathrm{NaCl}, 10 \%$ glycerol). The cells were lysed by sonication after a 0.5 h treatment with lysozyme (MilliporeSigma, Burlington, MA), benzonase nuclease (MilliporeSigma), and 1 cOmplete EDTAfree Protease Inhibitor Cocktail (MilliporeSigma). The clarified lysate was loaded onto a $1 \mathrm{~mL}$ HisTrap Fast Flow column (Cytiva, Marlborough, MA) at a flow rate of $1 \mathrm{~mL} / \mathrm{min}$. The column was washed with 100 column volumes (CV) of Buffer 1 $+50 \mathrm{mM}$ imidazole and pure protein eluted in $20 \mathrm{CV}$ Buffer $1+250 \mathrm{mM}$ imidazole. Fractions containing pure protein were buffer exchanged using PD-10 desalting columns (Cytiva) into storage buffer (50 mM phosphate $\mathrm{pH} 8.0,500 \mathrm{mM} \mathrm{NaCl}, 10 \%$ glycerol), aliquoted, and frozen at $-80^{\circ} \mathrm{C}$.

\section{B: Printing of protein arrays}

All protein arrays were printed using a Scienion sciFLEXARRAYER S12 instrument, following general instrument parameters described previously (8). Proteins were aspirated from a 384-well source plate (Scienion) maintained at dew point, and 
dispensed onto Fluotrac ${ }^{\mathrm{TM}}$ 600, Greiner Bio-One 96-well plates (Fisher Scientific, Waltham, MA) maintained at $60 \%$ humidity and ambient temperature, with drop volumes of 330-350 pl and 2 drops per spot. Coronavirus Spike, RBD and Nucleocapsid proteins were printed in either a 6x6 grid or 8x6 "checkerboard" pattern. Additional biotinylated anti-kappa light chain spots, GFP foldon spots, and anti-IgG Fc spots were also printed as fiducials, negative controls, and positive controls, respectively. The printed arrays were maintained at $75 \%$ relative humidity and ambient room temperature overnight to allow for adsorption of the proteins to the surface of the plate, followed by vacuum sealing and storage at $4^{\circ} \mathrm{C}$ until use.

\section{C: Open plate reader, Nautilus}

The plate reader, nick-named Nautilus, was derived from the open microscopy platform squid (34). The scanning unit was adapted to hold 96-well plates and an LED array, diffuser, and $650 \mathrm{~nm}$ filter were added to evenly illuminate the wells. The imaging setup consists of a 4x/0.1NA objective (Boli Optics), an $\mathrm{f}=35 \mathrm{~mm}$ imaging lens (Hivision) as tube lens and an industrial camera with monochrome Sony IMX226 CMOS sensor (Daheng). While motorized autofocus has been implemented, the data is collected with an early version of the system where manual focus is performed for well A1 before automated scanning of the entire plate starts. Images are saved as 8-bit bmp files for downstream processing. The system is controlled with a python-based graphical user interface, which is part of the squid framework and can be further integrated with the pysero processing pipeline.

\section{D: Multiplex ELISA analysis with pysero}

The source code for pysero is available at https://github.com/czbiohub/pysero

\section{Detection of spots}

Given a printed microarray with known fiducial and sample grid coordinate locations, the pysero spot detection pipeline will extract those locations from the metadata.xlsx file as well as other critical parameters such as number of rows, columns, vertical and horizontal pitch, spot width and pixel size. Spot detection is a multi-stage process beginning with several preprocessing steps to assist detection when the sample can be dirty or noisy. First, pysero extracts the inner-well area from the image using a combination of multi-modal intensity thresholding such as "otsu" and "rosin" thresholds, then checking that the area meets minimum size and eccentricity constraints. From this cropped image, we apply a uniform 2D Laplacian of Gaussian filter to the whole image using a kernel size sigma that is a quarter the spot width in nanometers. Next, this filtered image is fed into the openCV module SimpleBlobDetector (44). The module generates a range of binarized images from thresholds based on minimum and maximum intensity, groups blob center coordinates across binary images, and we filter out relevant blobs using the SimpleBlobDetector parameters minimum circularity, convexity, distance between spots, and repeatability. The outputted coordinates of proposed spots are further filtered based on distance from the border of the image.

The final step is to align the detected spots to the known printed array configuration of fiducials as defined in the metadata. Proposed spots may contain all spots in the array but may have missed some due to artifacts or lack of signal, and may contain false positives from noise. In order to find a robust fit between fiducial locations and proposed spots we use particle filtering (45), a sequential importance sampling method often used in tracking but can also be found in medical image segmentation applications (46). A particle is defined as a grid pose estimate, and we allow for translation, rotation, and scaling transformations when sampling particles. An initial grid estimate is based on the known physical size of the grid and is placed at the center of the image with 0 degrees of rotation and a scale of 1 . A random set of $\mathrm{N}=4000$ particles is generated and for each particle the sum of distances from fiducials to their nearest proposed spots is calculated. Weights are computed as the inverse of the summed distances and a new set of $\mathrm{N}$ particles is generated using importance sampling of these weights, with a small number of distortions added. This process allows for successful particles to proliferate while unsuccessful particles die out, and is repeated until convergence.

\section{Measurement of $O D$}

Once the fiducials of the array are identified using particle filtering, the expected positions of other spots can be determined by their positions on the grid. A simple threshold at $75 \%$ of pixel intensity level is performed within a bounding box around the expected spot position to segment the spot. The bounding box dimension is set to be twice as big as the expected spot 
width to account for spot printing and fiducial fitting errors. If no spot is detected within the bounding box, a circular mask with the expected spot size and position is used to compute the spot intensity. To estimate the background of the image, the image is first divided into blocks of $128 \times 128$ pixels. The median of each block is computed, and a second order 2D polynomial function is fit to the blocked median to get the background image. The spot and background intensities are then defined by the median pixel intensity of the sample and background images within the segmented spot. The OD value of a spot is defined by

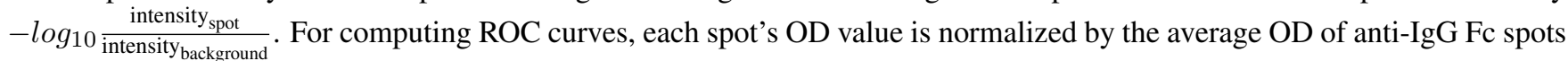
over the entire plate. OD values from the same antigen in the same well are then averaged. ROC curves and the area under the curve are computed using scikit-learn functions roc_curve and roc_auc_score. 95\% confidence intervals of the ROC curves are computed by stratified bootstrapping the OD values, which are then used to generate distributions of ROC curves.

\section{Classification model}

The normalized OD values of each antigen, anti-IgG Fc, and GFP foldon spots in each well were used to construct the feature vector of each serum sample. Binary gradient boosting tree classifiers are built and trained using xgboost (47) to distinguish the positive and negative samples. $60 \%$ of serum data $(\mathrm{N}=318)$ were used to train the model. Model training and hyperparameters tuning were done by choosing the model with the best average validation AUC score from 4-fold cross-validation (75\% training and $25 \%$ validation splits). The model performance was evaluated on the remaining $40 \%$ of the dataset (test set, $\mathrm{N}=189$ ) that were not used for training and model selection.

\section{E: Validation of multiSero with COVID-19 sera SARS-CoV-2 ELISA Array Assay}

Plasma samples were assayed with the SARS-CoV-2 ELISA array as described in Waltari et al. (8) with modified parameters. Briefly, $200 \mathrm{uL}$ blocking buffer $(0.05 \%$ Tween, $0.5 \%$ bovine serum albumin fraction $\mathrm{V}, 2 \%$ filtered fetal bovine serum, $0.2 \%$ bovine gamma-globulin, 0.05\% Proclin300TM, 5mM EDTA in PBS) was added to each well of the printed and cured ELISA array plates and allowed to incubate for 1 hour, then aspirated. $100 \mathrm{uL}$ sample serum diluted in blocking buffer was added to each well and allowed to incubate 1 hour. Sample was aspirated and wells incubated for 1 hour with $100 \mathrm{uL}$ of an even mixture of biotinylated goat antibodies targeting the kappa $\left(25 \mathrm{ng} \mathrm{mL}^{-1}\right.$, Southern Biotech cat. 2070-08) and lambda (25 ng $\mathrm{mL}^{-1}$ Southern Biotech cat. 2060-08) light chains. Wells were aspirated and incubated for one hour with $100 \mathrm{uL}$ streptavidinconjugated HRP $\left(0.2 \mu \mathrm{g} \mathrm{mL}{ }^{-1}\right)$. Wells were aspirated and developed with $50 \mathrm{uL}$ sciCOLOR T2 precipitating TMB reagent (Scienion). Wells were aspirated, sealed, and imaged shortly thereafter on a sciREADER CL2 plate reader (Scienion) or Nautilus plate reader. Wells were washed between steps with PBS 0.05\% Tween.

\section{SARS-CoV-2 positive samples and negative controls}

The SARS-CoV-2 ELISA array assay was validated using plasma samples from RT-PCR confirmed SARS-CoV-2 infected patients from the Long-term Impact of Infection With Novel Coronavirus (COVID-19) (LIINC, NCT04362150) study. Various plasma samples collected before the COVID-19 pandemic were used as negative controls. All samples were stored at $4^{\circ} \mathrm{C}$ and diluted 1:1 in HEPES buffer (40\% glycerol, 0.04\% NaN3, 40 mM HEPES in PBS), and further diluted 100X in ELISA array blocking buffer before assays. Pooled SARS-CoV-2 positive samples and CR3022 monoclonal mouse antibody synthesized in-house (48) were serially diluted to titrate antibody concentrations in samples run on the same ELISA array plate.

\section{Data Availability}

The images of SARS-CoV-2 ELISA-array assayed with 189 sera, spread over 6 plates, are available publicly via google drive. The python code to analyze this data is available from this GitHub repository. The repository also includes code and instructions to reproduce fig. 4 from above images.

\section{Acknowledgements}

Some of the illustrations shown in fig. 1, fig. 3, and fig. S3 were created with BioRender.com. 


\section{Funding}

J.R.B, E.W, S-M.G, J.F, B.B.C, I.E.I, M.L.F, W.W, C.M.T, K.M.M, M.P, B.G, J.E.P, S.B.M are supported by the Chan Zuckerberg Biohub. M.L.F was also supported by the Knight-Hennessy Scholarship and a Graduate Research Fellowship from the National Science Foundation. H.L was supported by Bio-X Stanford Interdisciplinary Graduate Fellowship. This research was supported by the Chan Zuckerberg Biohub and NIH/NIAID 3R01AI141003-03S1 (to TJ Henrich).

\section{Competing Interests}

The authors have declared no competing interest.

\section{Author contributions}

J.R.B, E.W, O. J, S-M.G, J.V, and A.D.L designed, performed, and analyzed the experiments in supervision of B.G, J.E.P, C.M.T, and S.B.M. S-M.G, J.F, B.B.C, and S.B.M developed analytical pipeline. E.W, K.M.M, and J.E.P developed the ELISAarray, and J.E.P and W.W performed protein synthesis. H.L, I.E.I, and M.L.F designed and built the plate reader in supervision of M. P. and S.B.M., and collected data with it. M.J.P, T.J.H, S.G.D, and B.G organized the LIINC study and guided analysis. J.R.B, E.W, O.J, S-M.G, B.B.C, J.E.P, and S.B.M. wrote the paper with inputs from all authors.

\section{References}

1. Xu Z, Shi L, Wang Y, Zhang J, Huang L, Zhang C, et al. Pathological Findings of COVID-19 Associated with Acute Respiratory Distress Syndrome. The Lancet Respiratory Medicine. 2020 Apr;8(4):420-422.

2. Rosenberg ES, Tesoriero JM, Rosenthal EM, Chung R, Barranco MA, Styer LM, et al. Cumulative Incidence and Diagnosis of SARS-CoV-2 Infection in New York. Annals of Epidemiology. 2020 Aug;48:23-29.e4

3. Hallal P, Hartwig F, Horta B, Victora GD, Silveira M, Struchiner C, et al. Remarkable Variability in SARS-CoV-2 Antibodies across Brazilian Regions: Nationwide Serological Household Survey in 27 States. medRxiv. 2020 May:2020.05.30.20117531.

4. Pollán M, Pérez-Gómez B, Pastor-Barriuso R, Oteo J, Hernán MA, Pérez-Olmeda M, et al. Prevalence of SARS-CoV-2 in Spain (ENE-COVID): A Nationwide, Population-Based Seroepidemiological Study. The Lancet. 2020 Aug;396(10250):535-544.

5. Merkely B, Szabó AJ, Kosztin A, Berényi E, Sebestyén A, Lengyel C, et al. Novel Coronavirus Epidemic in the Hungarian Population, a Cross-Sectional Nationwide Survey to Support the Exit Policy in Hungary. GeroScience. 2020 Aug;42(4):1063-1074.

6. Krammer F, Simon V. Serology Assays to Manage COVID-19. Science. 2020 Jun;368(6495):1060-1061.

7. Arora RK, Joseph A, Wyk JV, Rocco S, Atmaja A, May E, et al. SeroTracker: A Global SARS-CoV-2 Seroprevalence Dashboard. The Lancet Infectious Diseases. 2020 Aug;0(0).

8. Waltari E, Carabajal E, Sanyal M, Friedland N, McCutcheon KM. Adaption of a Conventional ELISA to a 96-Well ELISA-Array for Measuring the Antibody Responses to Influenza Virus Proteins and Vaccines. Journal of Immunological Methods. 2020 Jun;481-482:112789.

9. Wang D, Zheng Y, Kang X, Zhang X, Hao H, Chen W, et al. A Multiplex ELISA-Based Protein Array for Screening Diagnostic Antigens and Diagnosis of Flaviviridae Infection. European Journal of Clinical Microbiology \& Infectious Diseases: Official Publication of the European Society of Clinical Microbiology. 2015 Jul;34(7):1327-1336.

10. Aznar I, Frankena K, More SJ, Whelan C, Martin W, Gormley E, et al. Optimising and Evaluating the Characteristics of a Multiple Antigen ELISA for Detection of Mycobacterium Bovis Infection in a Badger Vaccine Field Trial. PLoS ONE. 2014 Jul;9(7):e100139.

11. Zamecnik CR, Rajan JV, Yamauchi KA, Mann SA, Loudermilk RP, Sowa GM, et al. ReScan, a Multiplex Diagnostic Pipeline, Pans Human Sera for SARS-CoV-2 Antigens. Cell Reports Medicine. 2020 Oct; $1(7): 100123$

12. Jin Y, Kim EM, Choi MH, Oh Md, Hong ST. Significance of Serology by Multi-Antigen ELISA for Tissue Helminthiases in Korea. Journal of Korean Medical Science. 2017 Jul;32(7):1118-1123.

13. Kang X, Li Y, Fan L, Lin F, Wei J, Zhu X, et al. Development of an ELISA-Array for Simultaneous Detection of Five Encephalitis Viruses. Virology Journal. 2012 Feb;9(1):56.

14. Lyashchenko KP, Singh M, Colangeli R, Gennaro ML. A Multi-Antigen Print Immunoassay for the Development of Serological Diagnosis of Infectious Diseases. Journal of Immunological Methods. 2000 Aug;242(1-2):91-100.

15. Zhang SL, Zhao JW, Sun ZQ, Yang EZ, Yan JH, Zhao Q, et al. Development and Evaluation of a Novel Multiple-Antigen ELISA for Serodiagnosis of Tuberculosis. Tuberculosis. 2009 Jul;89(4):278284.

16. Shete PB, Ravindran R, Chang E, Worodria W, Chaisson LH, Andama A, et al. Evaluation of Antibody Responses to Panels of M. Tuberculosis Antigens as a Screening Tool for Active Tuberculosis in Uganda. PLOS ONE. 2017 Aug;12(8):e0180122.

17. Khaliq A, Ravindran R, Hussainy SF, Krishnan VV, Ambreen A, Yusuf NW, et al. Field Evaluation of a Blood Based Test for Active Tuberculosis in Endemic Settings. PLOS ONE. 2017 Apr;12(4):e0173359.

18. Menon S, Stansfield SH, Logan B, Hocking JS, Timms P, Rombauts L, et al. Development and Evaluation of a Multi-Antigen Peptide ELISA for the Diagnosis of Chlamydia Trachomatis-Related Infertility in Women. Journal of Medical Microbiology. 2016 Sep;65(9):915-922.

19. Hara Y, Chin CY, Mohamed R, Puthucheary SD, Nathan S. Multiple-Antigen ELISA for Melioidosis - a Novel Approach to the Improved Serodiagnosis of Melioidosis. BMC Infectious Diseases. 2013 Apr;13:165.

20. Long QX, Liu BZ, Deng HJ, Wu GC, Deng K, Chen YK, et al. Antibody Responses to SARS-CoV-2 in Patients with COVID-19. Nature Medicine. 2020 Jun;26(6):845-848.

21. Long QX, Tang XJ, Shi QL, Li Q, Deng HJ, Yuan J, et al. Clinical and Immunological Assessment of Asymptomatic SARS-CoV-2 Infections. Nature Medicine. 2020 Aug;26(8):1200-1204.

22. Wajnberg A, Amanat F, Firpo A, Altman D, Bailey M, Mansour M, et al. SARS-CoV-2 Infection Induces Robust, Neutralizing Antibody Responses That Are Stable for at Least Three Months. Infectious Diseases (except HIV/AIDS); 2020

23. Elders PND, Dhawan S, Tanganuchitcharnchai A, Phommasone K, Chansamouth V, Day NPJ, et al. Diagnostic Accuracy of an In-House Scrub Typhus Enzyme Linked Immunoassay for the Detection of IgM and IgG Antibodies in Laos. PLOS Neglected Tropical Diseases. 2020 Dec;14(12):e0008858.

24. de Oliveira IQ, Silva RA, Sucupira MV, da Silva ED, Reis AB, Grimaldi G, et al. Multi-Antigen Print Immunoassay (MAPIA)-Based Evaluation of Novel Recombinant Leishmania Infantum Antigens for the Serodiagnosis of Canine Visceral Leishmaniasis. Parasites \& Vectors. 2015 Jan;8(1):45. 
medRxiv preprint doi: https://doi.org/10.1101/2021.05.07.21249238; this version posted May 11, 2021. The copyright holder for this preprint

(which was not certified by peer review) is the author/funder, who has granted medRxiv a license to display the preprint in perpetuity.

It is made available under a CC-BY 4.0 International license .

5. Erikson JM, Valente AJ, Mummidi S, Kandikattu HK, DeMarco VG, Bender SB, et al. Targeting TRAF3IP2 by Genetic and Interventional Approaches Inhibits Ischemia/Reperfusion-Induced Myocardial Injury and Adverse Remodeling. Journal of Biological Chemistry. 2017 Feb;292(6):2345-2358.

26. Himburg HA, Doan PL, Quarmyne M, Yan X, Sasine J, Zhao L, et al. Dickkopf-1 Promotes Hematopoietic Regeneration via Direct and Niche-Mediated Mechanisms. Nature medicine. 2017 Jan;23(1):91-99.

27. Embers ME, Hasenkampf NR, Barnes MB, Didier ES, Philipp MT, Tardo AC. Five-Antigen Fluorescent Bead-Based Assay for Diagnosis of Lyme Disease. Clinical and Vaccine Immunology : CVI. 2016 Apr;23(4):294-303.

28. Lebani K, Jones ML, Watterson D, Ranzoni A, Traves RJ, Young PR, et al. Isolation of Serotype-Specific Antibodies against Dengue Virus Non-Structural Protein 1 Using Phage Display and Application in a Multiplexed Serotyping Assay. PLoS ONE. 2017 Jul;12(7).

29. Michlmayr D, Kim EY, Rahman AH, Raghunathan R, Kim-Schulze S, Che Y, et al. Comprehensive Immunoprofiling of Pediatric Zika Reveals Key Role for Monocytes in the Acute Phase and No Effect of Prior Dengue Virus Infection. Cell Reports. 2020 Apr;31(4).

30. Drouot L, Hantz S, Jouen F, Velay A, Lamia B, Veber B, et al. Evaluation of Humoral Immunity to SARS-CoV-2: Diagnostic Value of a New Multiplex Addressable Laser Bead Immunoassay. Frontiers in Microbiology. 2020;11:603931.

31. Tiwari RP, Jain A, Khan Z, Kumar P, Bhrigu V, Bisen PS. Designing of Novel Antigenic Peptide Cocktail for the Detection of Antibodies to HIV-1/2 by ELISA. Journal of Immunological Methods. 2013 Jan;387(1-2):157-166

32. Moendeg KJ, Angeles JMM, Goto Y, Leonardo LR, Kirinoki M, Villacorte EA, et al. Development and Optimization of Cocktail-ELISA for a Unified Surveillance of Zoonotic Schistosomiasis in Multiple Host Species. Parasitology Research. 2015 Mar;114(3):1225-1228.

33. Bolton JS, Chaudhury S, Dutta S, Gregory S, Locke E, Pierson T, et al. Comparison of ELISA with Electro-Chemiluminescence Technology for the Qualitative and Quantitative Assessment of Serological Responses to Vaccination. Malaria Journal. 2020 Apr;19.

34. Li H, Krishnamurthy D, Li E, Vyas P, Akireddy N, Chai C, et al. Squid: Simplifying Quantitative Imaging Platform Development and Deployment. bioRxiv. 2020 Dec:2020.12.28.424613.

35. Liu W, Liu L, Kou G, Zheng Y, Ding Y, Ni W, et al. Evaluation of Nucleocapsid and Spike Protein-Based Enzyme-Linked Immunosorbent Assays for Detecting Antibodies against SARS-CoV-2. Journal of Clinical Microbiology. 2020 May;58(6).

36. Tehrani ZR, Saadat S, Saleh E, Ouyang X, Constantine N, DeVico AL, et al. Specificity and Performance of Nucleocapsid and Spike-Based SARS-CoV-2 Serologic Assays. medRxiv.

37. Amanat F, Nguyen T, Chromikova V, Strohmeier S, Stadlbauer D, Javier A, et al. A Serological Assay to Detect SARS-CoV-2 Seroconversion in Humans. medRxiv. 2020 Mar:2020.03.17.20037713.

38. Tian X, Li C, Huang A, Xia S, Lu S, Shi Z, et al. Potent Binding of 2019 Novel Coronavirus Spike Protein by a SARS Coronavirus-Specific Human Monoclonal Antibody. Emerging Microbes \& Infections. 2020;9(1):382-385.

39. Tadesse LF, Safir F, Ho CS, Hasbach X, Khuri-Yakub BP, Jeffrey SS, et al. Toward Rapid Infectious Disease Diagnosis with Advances in Surface-Enhanced Raman Spectroscopy. The Journal of Chemical Physics. 2020 Jun;152(24):240902.

40. Liu H, Crooks RM. Three-Dimensional Paper Microfluidic Devices Assembled Using the Principles of Origami. Journal of the American Chemical Society. 2011 Nov;133(44):17564-17566.

41. Sanjay ST, Dou M, Sun J, Li X. A Paper/Polymer Hybrid Microfluidic Microplate for Rapid Quantitative Detection of Multiple Disease Biomarkers. Scientific Reports. 2016 Jul;6.

42. Carrilho E, Phillips ST, Vella SJ, Martinez AW, Whitesides GM. Paper Microzone Plates. Analytical Chemistry. 2009 Aug;81(15):5990-5998.

43. Pilarowski G, Lebel P, Sunshine S, Liu J, Crawford E, Marquez C, et al. Performance Characteristics of a Rapid SARS-CoV-2 Antigen Detection Assay at a Public Plaza Testing Site in San Francisco. medRxiv. 2020 Jan:2020.11.02.20223891.

44. Bradski J. The OpenCV Library; 2000 .

45. Doucet A, Johansen AM. A Tutorial on Particle Filtering and Smoothing: Fifteen Years Later; 2011.

46. de Bruijne M, Nielsen M. Shape Particle Filtering for Image Segmentation. In: Barillot C, Haynor DR, Hellier P, editors. Medical Image Computing and Computer-Assisted Intervention - MICCAI 2004. Lecture Notes in Computer Science. Berlin, Heidelberg: Springer; 2004. p. 168-175.

47. Chen T, Guestrin C. XGBoost: A Scalable Tree Boosting System. In: Proceedings of the 22nd ACM SIGKDD International Conference on Knowledge Discovery and Data Mining. KDD '16. New York, NY, USA: Association for Computing Machinery; 2016. p. 785-794.

48. Powell AE, Zhang K, Sanyal M, Tang S, Weidenbacher PA, Li S, et al. A Single Immunization with Spike-Functionalized Ferritin Vaccines Elicits Neutralizing Antibody Responses against SARS-CoV-2 in Mice. bioRxiv. 2020 Aug:2020.08.28.272518. 
medRxiv preprint doi: https://doi.org/10.1101/2021.05.07.21249238; this version posted May 11, 2021. The copyright holder for this preprint (which was not certified by peer review) is the author/funder, who has granted medRxiv a license to display the preprint in perpetuity.

It is made available under a CC-BY 4.0 International license .

\section{Supplementary Figures}
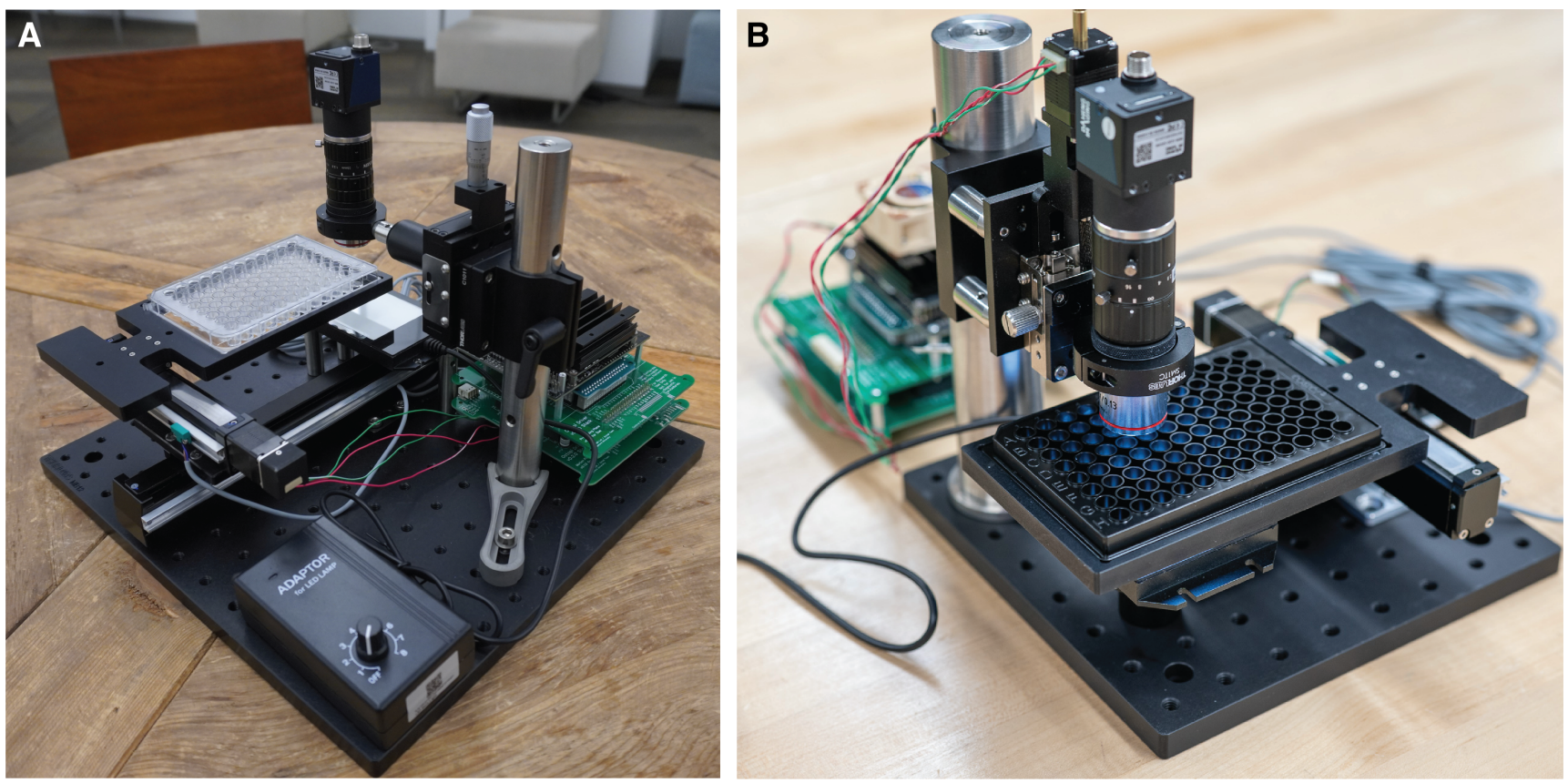

Fig. S1. Photos of Squid plate reader (Nautilus). (A) Photo of the original unit used for data collection. (B) Photo of the current version that has motorized focus adjustment. 
medRxiv preprint doi: https://doi.org/10.1101/2021.05.07.21249238; this version posted May 11, 2021. The copyright holder for this preprint (which was not certified by peer review) is the author/funder, who has granted medRxiv a license to display the preprint in perpetuity.

It is made available under a CC-BY 4.0 International license .

\section{Tables}

\begin{tabular}{|c|c|c|c|c|c|c|}
\hline \# & Description & Vendor & Part Number / File for CNC machining & Qty & Unit Price & Image Reference \\
\hline & Motorized Focus Assembly & & & & & \\
\hline 1 & ball bearing linear stage, stainless steel, $\pm 6.5 \mathrm{~mm}$ & dg-sl & LBX40-C (product page) & 1 & $\$ 61.14$ & \\
\hline 2 & adapter for mounting SM1TC to LBX40-C & XTJ-tech & adapter_LBX40_SM1TC(thick)_v2.zip & 1 & $\$ 25.00$ & \\
\hline 3 & adapter for mounting LBX40-C to C1511 & XTJ-tech & adapter_LBX40_C1511_v3.zip & 1 & $\$ 25.00$ & \\
\hline 4 & adapter for mounting $21 \mathrm{H} 4 \mathrm{U}$ to LBX40-C & XTJ-tech & adapter_LBX40_21H4U_v3.stp & 1 & $\$ 55.00$ & \\
\hline 5 & size 8 captive linear actuator, $6 e-5$ in/step & Haydon kerk & 21H4U-2.5-A98 v1 (based on 21H4U-2.5-907) & 1 & $\$ 130.45$ & \\
\hline 7 & Clamp for SM1 Lens Tubes & Thorlabs & SM1TC & 1 & $\$ 45.72$ & \\
\hline 8 & SM1 Lens Tube, 0.3" threaded depth & Thorlabs & SM1L03 & 1 & $\$ 12.52$ & \\
\hline 9 & SM1 adapter for Objectives with RMS thread (Olympus, & , Thorlabs & SM1A3 & 1 & $\$ 18.50$ & \\
\hline 11 & M3 $\times 15 \mathrm{~mm}$ (or $14 \mathrm{~mm}$ ) socket head screws for mountil i & i McMaster-Carr & $91292 A 346$ or $91292 A 027$ & 4 & & \\
\hline 12 & M3 $\times 5 \mathrm{~mm}$ socket head screws for mounting the LBX4C & CMcMaster-Carr & 91292A110 & 4 & & \\
\hline 13 & $8-32 \times 1 / 2$ " socket head screws for mounting the assem & McMaster-Carr & 92196A194 & 4 & & \\
\hline 14 & 8-32 $\times 1 / 4$ " socket head screws for mounting SM1TC & McMaster-Carr & 92196A190 & 1 & & \\
\hline 15 & M2.5 $\times 8 \mathrm{~mm}$ socket head screws for adapter mounting & McMaster-Carr & $91292 A 012$ & 2 & & \\
\hline 16 & M2 $\times 6 \mathrm{~mm}$ socket head screws for mounting the linear a & actuator & & 4 & & \\
\hline \multicolumn{7}{|c|}{ Total: $\$ 312.19$} \\
\hline & \multicolumn{2}{|c|}{ "Microscope Body" (may be replaced by custom machined blocks) } & & & & \\
\hline 1 & $\varnothing 1.5 "$ Post Mounting Clamp, 2.50" x 2.50" & Thorlabs & C1511 & 1 & $\$ 71.57$ & \\
\hline 2 & $\varnothing 1.5 "$ Mounting Post, $1 / 4 "-20$ Taps, $L=8$ " (other length & Thorlabs & P8 & 1 & $\$ 59.79$ & \\
\hline 3 & Studded Pedestal Base Adapter, 1/4"-20 Thread & Thorlabs & PB4 & 1 & $\$ 13.74$ & \\
\hline 4 & Clamping Fork for Ø1.5" Pedestal Post or Post Pedesta & Thorlabs & PF175B & 1 & $\$ 17.00$ & \\
\hline 5 & Aluminum Breadboard 6" x 6" x 1/2", 1/4"-20 Taps (othe & Thorlabs & MB6 & 1 & $\$ 51.48$ & \\
\hline 6 & Ø18.0 mm Sorbothane Feet, Adhesive Mounting Surfac & Thorlabs & AV3 & 1 & $\$ 19.91$ & \\
\hline 7 & 1/4-20 socket head screw and washer & & & 1 & & \\
\hline \multicolumn{7}{|c|}{ Total: $\$ 233.49$} \\
\hline & Imaging Lens and Camera Assembly & & & & & \\
\hline 1 & USB3 camera, Sony IMX 226, 1.85 um, $12 \mathrm{MP}, 32 \mathrm{fps}$ & Daheng & MER-1220-32U3M & 1 & $\$ 260.00$ & \\
\hline 3 & $f=50 \mathrm{~mm}$ machine vision lens $(1 / 1.8 ", f 2.4,10 \mathrm{MP}$ ratec & C HIKROBOT & MVL-HF5024M-10MP (distributor link, MTF) & 1 & $\$ 136.00$ & \\
\hline 5 & M27 ext - SM1 int adapter for MVL-HF5024M-10MP & Thorlabs & SM1A35 & 1 & $\$ 21.86$ & \\
\hline \multicolumn{7}{|c|}{ Total: $\$ 417.86$} \\
\hline & Alternative $140 \mathrm{~mm} \times$ (up to) $140 \mathrm{~mm}$ stage & & & & & \\
\hline 1 & motorized translation stage, $80 \mathrm{~mm}$ travel & Kgg-robot & \multicolumn{2}{|l|}{ SSME20-RO2-080L (being replaced by alternative from Kgg-robot ol 1} & $\$ 150-800, c$ & \\
\hline 2 & motorized translation stage, $140 \mathrm{~mm}$ travel & Kgg-robot & \multicolumn{2}{|l|}{ AVD30-RE18-200t (being replaced by alternative from Kgg-robot o। 1} & \multirow{2}{*}{$\$ 150-800, c$} & \\
\hline 6 & well plate holder (MIC-6 Al) & XTJ-tech & (being revised) & 1 & & \\
\hline 7 & well plate format glass slide holder (4 slots) & XTJ-tech & (being revised) & 0 & $\$ 18.00$ & \\
\hline \multicolumn{6}{|c|}{ (Total: $\$ 400-\$ 1000$, depending on options) } & \\
\hline & LED matrix illuminator & & & & & \\
\hline 1 & APA102-2020 8x8 RGB LED Grid & Adafruit & 3444 (to be replaced with custom made board) & 1 & $\$ 24.95$ & \\
\hline 2 & Cage Plate, for mounting the LED matrix & Thorlabs & CP37 & 1 & $\$ 19.91$ & \\
\hline 3 & Cage Plate, for condenser & Thorlabs & CP33 & 1 & $\$ 16.89$ & \\
\hline 4 & Aspheric Condenser Lens w/ Diffuser, Ø25 mm, $\mathrm{f}=20.1$ & | Thorlabs & ACL2520U-DG6-A & 1 & $\$ 30.84$ & \\
\hline 5 & Cage Rods 1" (pack of 4), for connecting the assembly & Thorlabs & ER1-P4 & 1 & $\$ 19.77$ & \\
\hline \multicolumn{7}{|c|}{ Total: $\$ 112.36$} \\
\hline & Objective & & & & & \\
\hline \multirow[t]{2}{*}{1} & $4 \mathrm{x} / 0.13$ ( 0.17 coverslip correction) Plan Fluor WD $16.3 \mathrm{~m}$ & I Boli Optics & FM13013231 & 1 & $\$ 88.98$ & \\
\hline & Filter & & & & & \\
\hline \multirow[t]{2}{*}{1} & $655 \mathrm{~nm} / 40 \mathrm{~nm}$ band pass filter & Semrock & FF02-655/40-25 & 0 & $\$ 345.00$ & \\
\hline & $655 \mathrm{~nm} / 30 \mathrm{~nm}$ band pass filter & Chroma & AT655/30m & 1 & $\$ 200.00$ & \\
\hline \multicolumn{6}{|c|}{ Total (without motorized XY stage): $\$ 1426.02$} & \\
\hline
\end{tabular}

Table S1. Nautilus Bill of Materials 
medRxiv preprint doi: https://doi.org/10.1101/2021.05.07.21249238; this version posted May 11, 2021. The copyright holder for this preprint (which was not certified by peer review) is the author/funder, who has granted medRxiv a license to display the preprint in perpetuity.
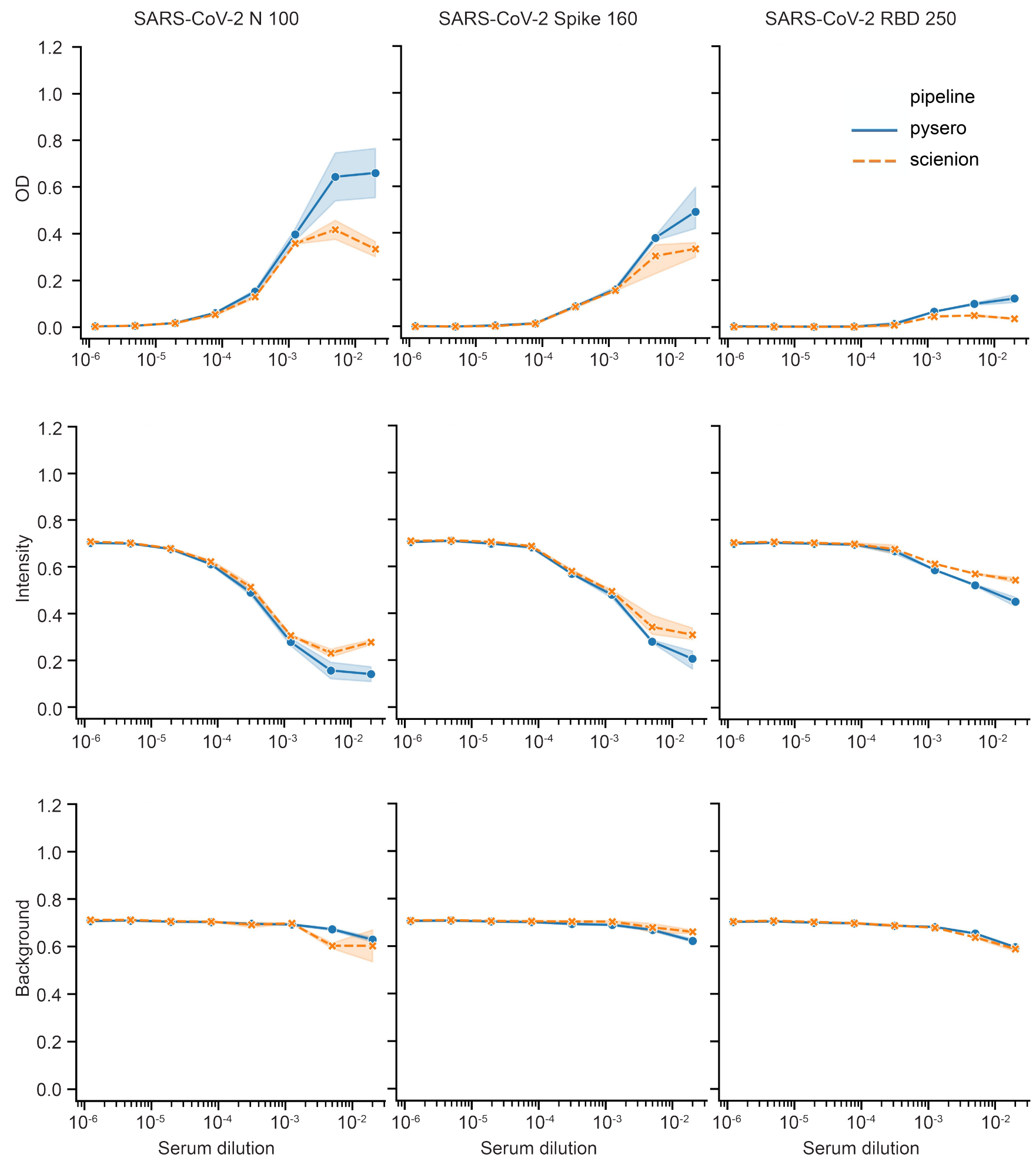

Fig. S2. Comparison of Scienion analysis platform and pysero. Images from a SciREADER CL2 were analyzed using either pysero (blue line) or Scienion analysis platform (orange dashed line). Analyzed OD (top), intensity (middle), and background intensity (bottom) for antibody resp\&nses of a single SARS-CoV-2, ppsitive serum to three antigens (left to right: SARS-CoV-2 N, Spike, RBD) are shown as a function of serum dilution. Shades around lines represent $95 \%$ confidence intervals around the mean of triplicate spots. 
medRxiv preprint doi: https://doi.org/10.1101/2021.05.07.21249238; this version posted May 11, 2021. The copyright holder for this preprint (which was not certified by peer review) is the author/funder, who has granted medRxiv a license to display the preprint in perpetuity.

It is made available under a CC-BY 4.0 International license.

A
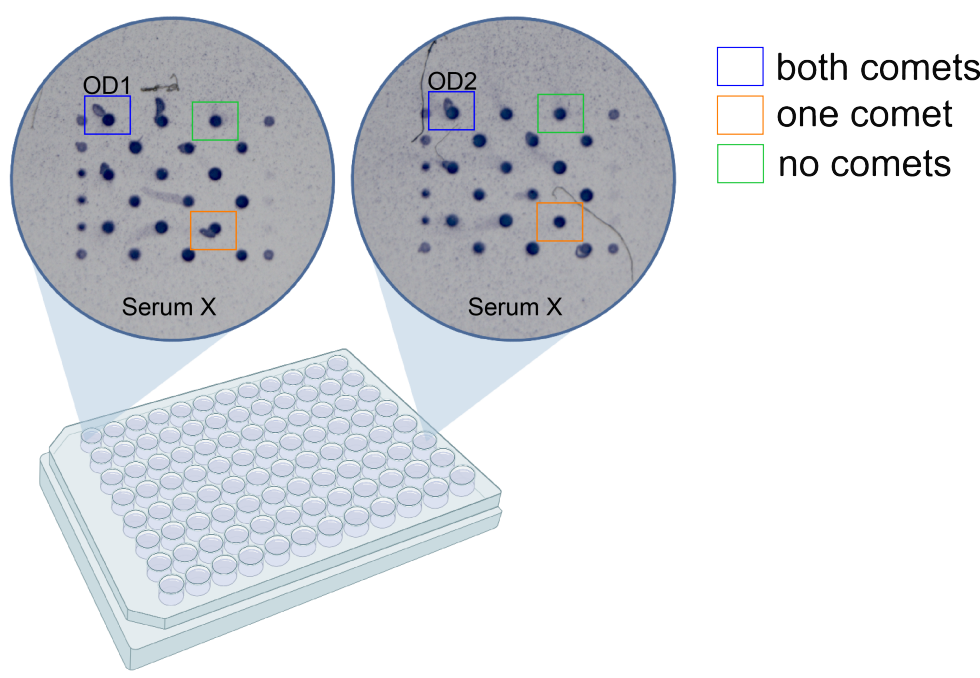

B

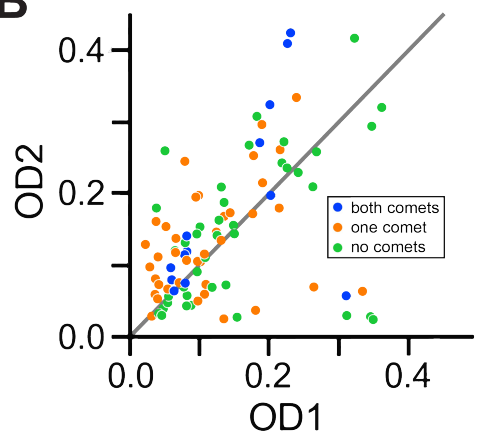

both comets

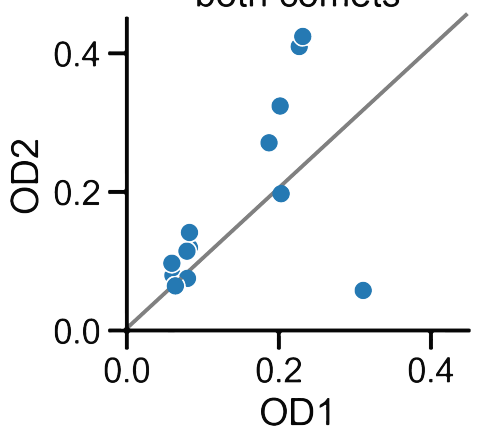

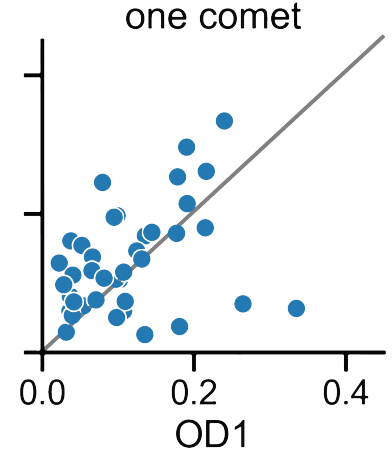

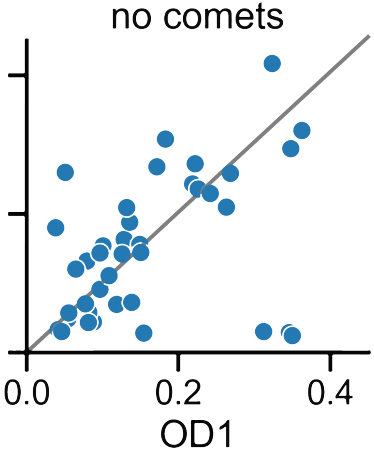

Fig. S3. Evaluation of how comets affect measured ODs using duplicate ELISA-array wells. ODs from spots at the same locations in the array grid were compared across duplicate wells. (A) Schematic of example spot-spot comparison. Top-left non-fiducial spot in well $A 1$ containing serum $X$ was compared to the top-left non-fiducial spot in well F12 also containing serum X. (B)The data for one plate of duplicate sera are split according to the number of comets in the spot pairs: spot pairs in which one spot had a comet (orange); spot pairs in which both spots had comets (blue); and spot pairs in which neither spot had a comet (green). A $y=x$ line is denoted on the plot (grey line). We find that the presence of comets does not add observable bias or variance to OD measurements. 
medRxiv preprint doi: https://doi.org/10.1101/2021.05.07.21249238; this version posted May 11, 2021. The copyright holder for this preprint (which was not certified by peer review) is the author/funder, who has granted medRxiv a license to display the preprint in perpetuity.
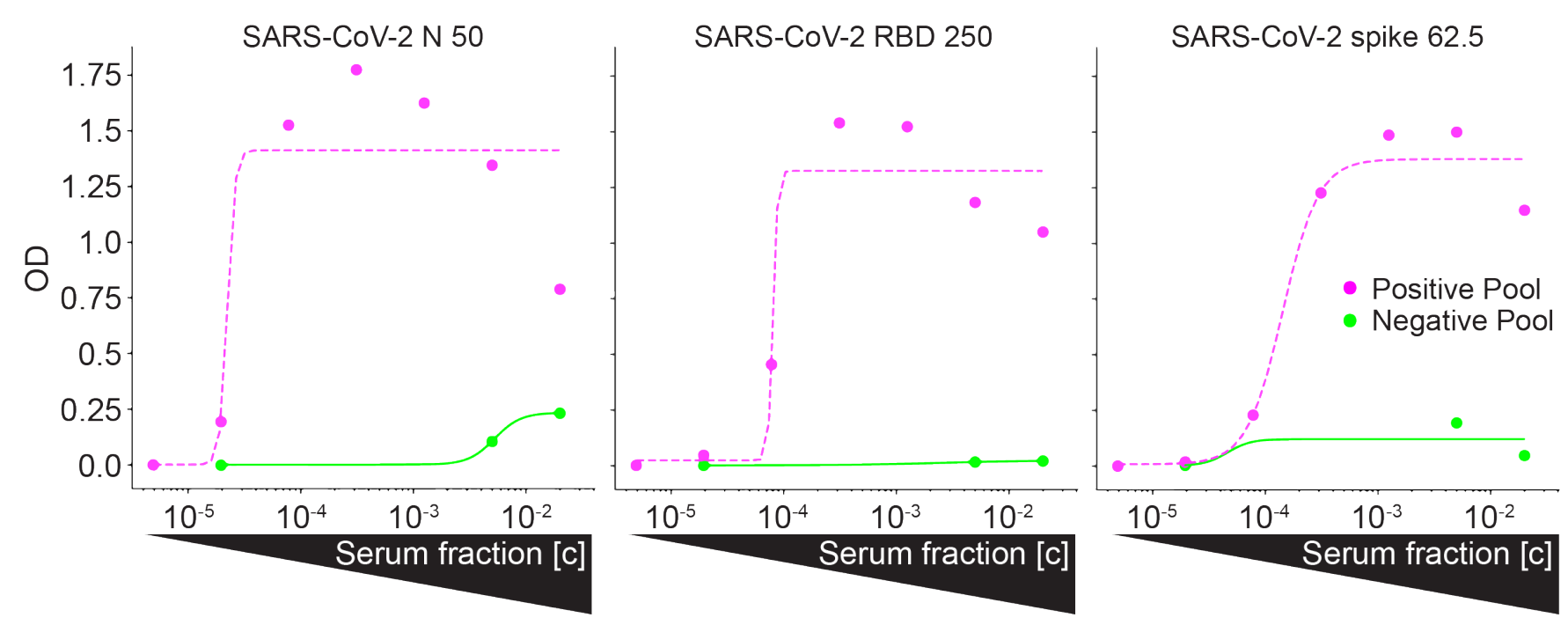

Fig. S4. Reactivity of Positive Pool and Negative Pool. After OD extraction from the registered grid coordinates, pysero generates plots according to user-specified parameters. Representative plots of antibody responses against the 3 SARS-CoV-2 antigens in the array: N (left), RBD (center), and spike (right). Positive Pool indicates pooled sera from SARS-CoV-2 RT-PCR-positive individuals (magenta points) and Negative Pool indicates pooled sera from individuals prior to the pandemic (green points). The respective curves are 4 parameter logistic regressions fit to $O D$ values of a serial dilutions of the sera, with diminished $O D$ values at highest concentrations likely due to the 'hook effect' (8). Pysero can output curve fits (shown above), categorical plots, and receiver operating characteristic analysis.

\begin{tabular}{|l|c|c|c|c|c|}
\hline Antigen & AUC & TPR 95\% Specificity & TPR 99\% Specificity & Mean +- sd OD of Neg Pool & Mean +- sd [Ab] of Neg Pool \\
\hline N 50 & 0.969 & $0.907(0.816-0.939)$ & $0.638(0.398-0.826)$ & $0.1047 \pm 0.02238$ & $0.0011 \pm 0.00055$ \\
\hline N 100 & 0.968 & $0.907(0.822-0.943)$ & $0.541(0.238-0.834)$ & $0.1087 \pm 0.02432$ & $0.0079 \pm 0.00036$ \\
\hline RBD 250 & 0.983 & $0.977(0.957-0.992)$ & $0.977(0.936-0.992)$ & $0.0160 \pm 0.01834$ & $0.0004 \pm 0.00025$ \\
\hline RBD 500 & 0.982 & $0.977(0.957-0.992)$ & $0.977(0.957-0.992)$ & $0.0230 \pm 0.00735$ & $*$ \\
\hline Spike 62.5 & 0.994 & $0.988(0.973-1)$ & $0.984(0.905-0.994)$ & $0.1933 \pm 0.04399$ & $0.0134 \pm 0.00266$ \\
\hline Spike 125 & 0.989 & $0.984(0.969-0.996)$ & $0.981(0.897-0.992)$ & $0.0927 \pm 0.01977$ & $*$ \\
\hline
\end{tabular}

Table S2. Summary statistics for antigens in ELISA-array. Area under the curve (AUC), sensitivity (true positive rate; TPR) at 95\% and 99\% specificity (N=507), mean $\mathrm{OD}$ and mean binding of Negative Pool expressed as relative concentration of mAb CR3022 standard curve in Negative Pool ([Ab] of Neg Pool; units are $\mu \mathrm{g}$ mL ${ }^{-1}$ ) for each antigen. Asterisks in the relative antibody concentration of Negative Pool column indicate that the mean OD of the Negative Pool for these antigens was lower than the lowest mAb CR3022 concentration in the standard curve. 
medRxiv preprint doi: https://doi.org/10.1101/2021.05.07.21249238; this version posted May 11, 2021. The copyright holder for this preprint (which was not certified by peer review) is the author/funder, who has granted medRxiv a license to display the preprint in perpetuity.

It is made available under a CC-BY 4.0 International license.
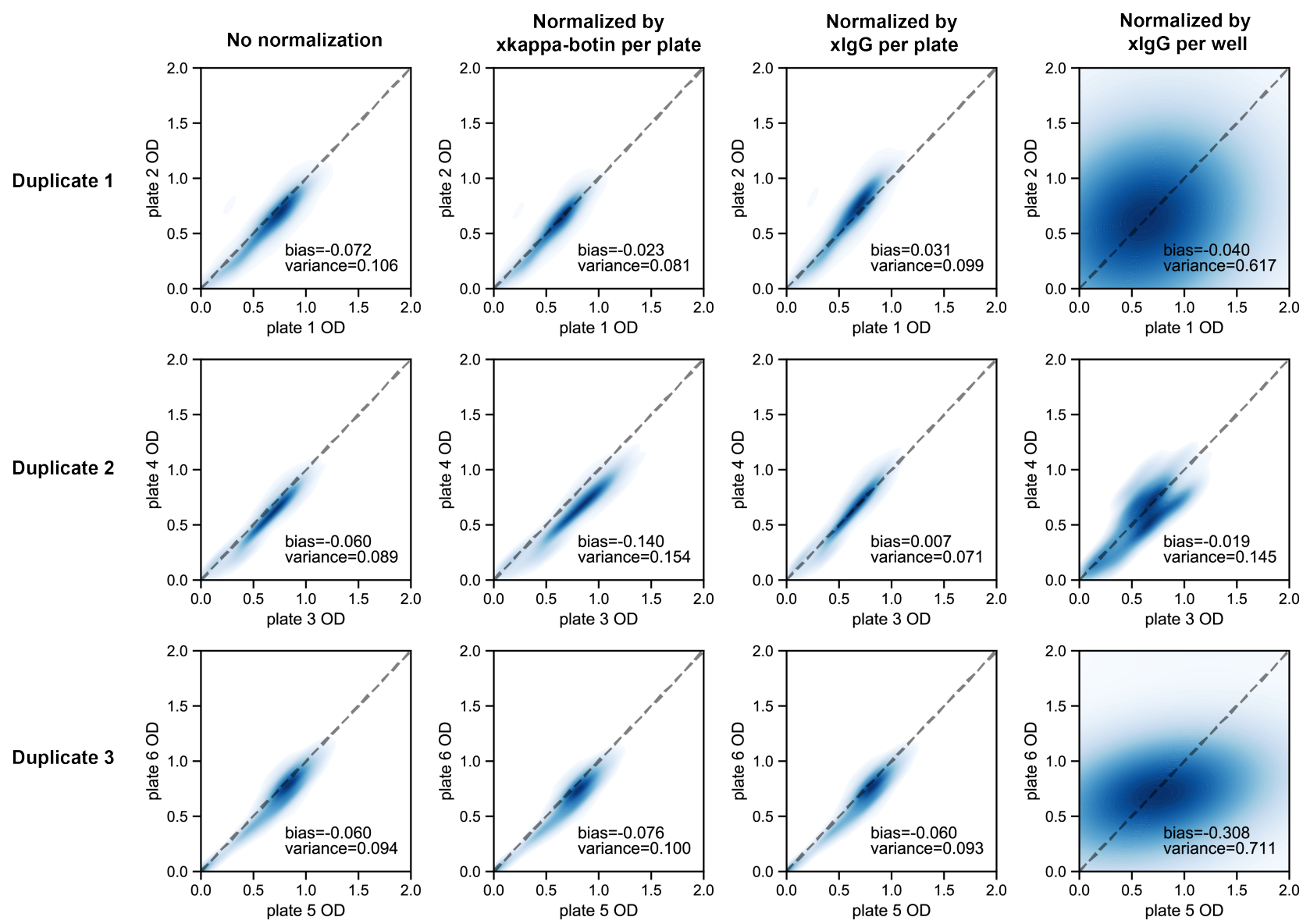

Fig. S5. Comparison of normalization methods for correcting biases and variances in ODs across plates. 2D distribution of duplicate OD values of antibody responses of RT-PCR positive sera to SARS-CoV-2 antigens. Spots with the same array location on duplicate plates are plotted against each other and then smoothed by the kerneldensity function to show the density of the data points (indicated by the brightness of the blue color, high brightness indicates low density). The spot OD values were normalized by dividing the mean of the reference spot ODs (anti-IgG Fc or fiducial spot) over each plate or well as indicated at the top. Duplicates with identical spot OD values will follow the function $y=x$ (dashed line). Performance of different normalization schemes are quantified by bias and variance of the normalized OD values across plates, which are defined by mean of $y-x$ and $|y-x| .3$ duplicates (6 plates) are shown. Normalizing ODs by the mean of anti-lgG Fc ODs over each plate consistently reduces bias and variance in all 3 duplicates. 
medRxiv preprint doi: https://doi.org/10.1101/2021.05.07.21249238; this version posted May 11, 2021. The copyright holder for this preprint (which was not certified by peer review) is the author/funder, who has granted medRxiv a license to display the preprint in perpetuity.

\section{A SciREADER CL2}

SARS-CoV-2 N 100

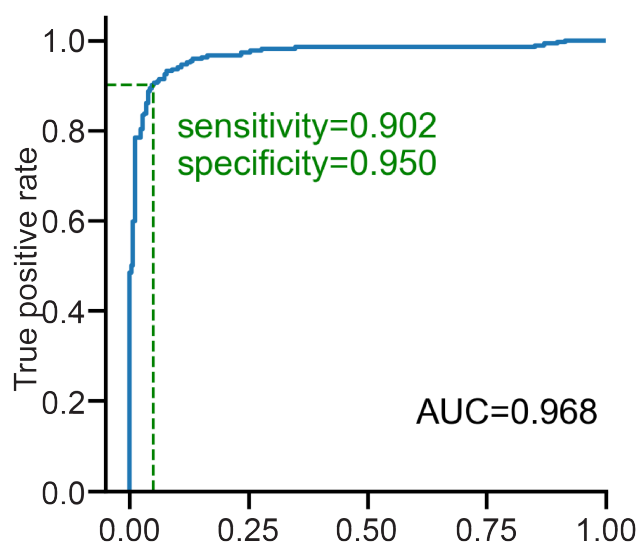

SARS-CoV-2 Spike 125

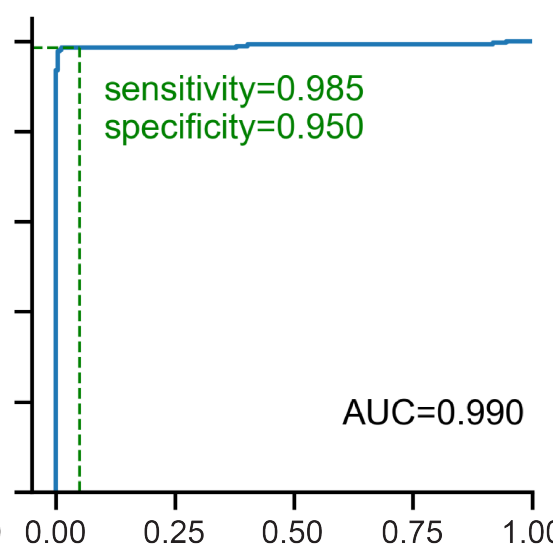

SARS-CoV-2 RBD 250

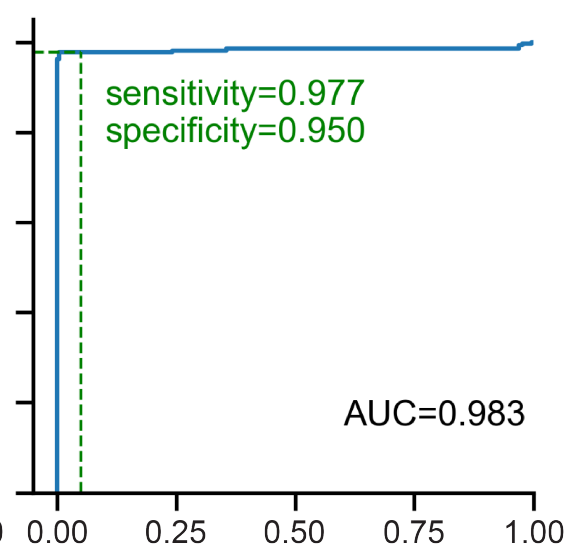

\section{B Nautilus}
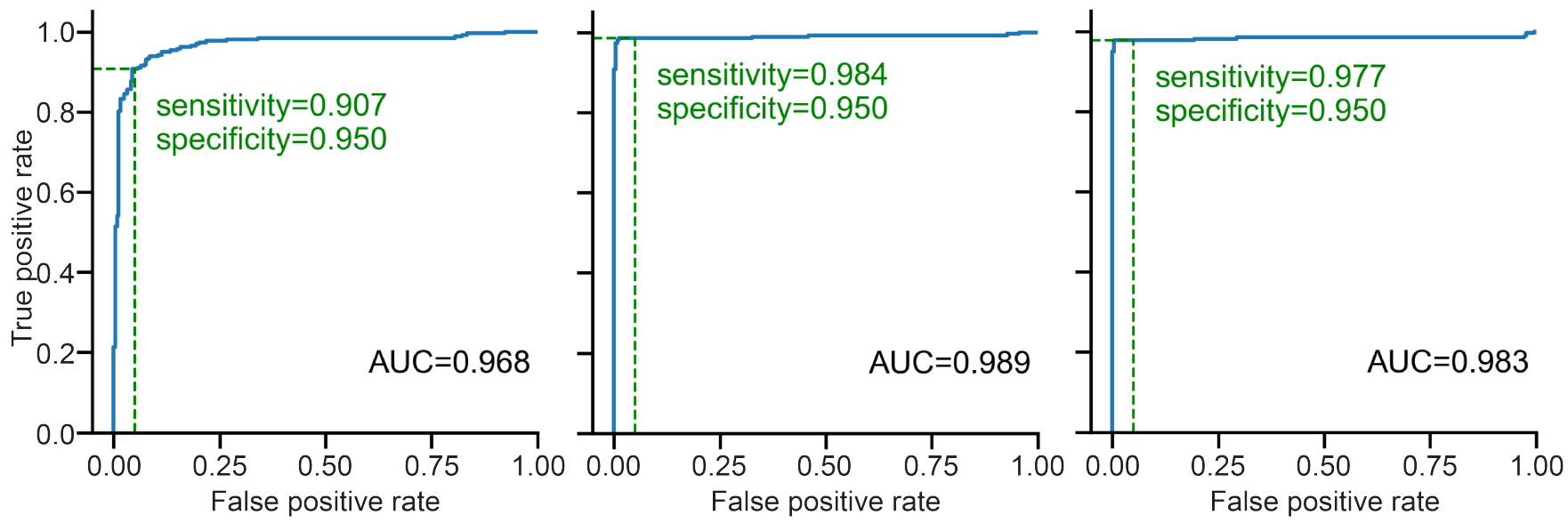

Fig. S6. Classification accuracy for images acquired with SciREADER CL2 and Nautilus, and analyzed with pysero. (A-B) ROC curves for single antigens (left to right: SARS-CoV-2 N, Spike, RBD) for array images acquired using SciREADER CL2 (top) or Nautilus (bottom). Images from each platform were analyzed using pysero. Blue shades represent $95 \%$ confidence intervals of ROC curves (blue line). For each antigen, the sensitivity (True positive rate) at $95 \%$ specificity (1 - false positive rate) is denoted (green dashed line) on the curve. 95\% confidence interval of area under the curve (AUC) is reported below each curve ( $\mathrm{N}=507)$. 